\title{
Triggering long-lived particles in HL-LHC and the challenges in the first stage of the trigger system
}

\author{
Biplob Bhattacherjee, ${ }^{a}$ Swagata Mukherjee, ${ }^{b}$ Rhitaja Sengupta ${ }^{a}$ and Prabhat Solanki ${ }^{a}$ \\ ${ }^{a}$ Centre for High Energy Physics, Indian Institute of Science, \\ C.V. Raman Road, Bangalore 560012, India \\ ${ }^{b}$ III. Physikalisches Institut A, RWTH Aachen University, \\ Otto-Blumenthal-Str. 16, Aachen 52074, Germany \\ E-mail: biplob@iisc.ac.in, mukherjee@physik.rwth-aachen.de, \\ rhitaja@iisc.ac.in, prabhats@iisc.ac.in
}

ABSTRACT: Triggering long-lived particles (LLPs) at the first stage of the trigger system is very crucial in LLP searches to ensure that we do not miss them at the very beginning. The future High Luminosity runs of the Large Hadron Collider will have increased number of pile-up events per bunch crossing. There will be major upgrades in hardware, firmware and software sides, like tracking at level-1 (L1). The L1 trigger menu will also be modified to cope with pile-up and maintain the sensitivity to physics processes. In our study we found that the usual level-1 triggers, mostly meant for triggering prompt particles, will not be very efficient for LLP searches in the 140 pile-up environment of HL-LHC, thus pointing to the need to include dedicated L1 triggers in the menu for LLPs. We consider the decay of the LLP into jets and develop dedicated jet triggers using the track information at L1 to select LLP events. We show in our work that these triggers give promising results in identifying LLP events with moderate trigger rates.

KeYwords: Jets, Phenomenological Models

ARXIV EPRINT: 2003.03943 


\section{Contents}

1 Introduction 1

2 The high luminosity LHC and triggering long-lived particles 4

2.1 The Phase-II HL-LHC upgrade and level-1 triggers 4

2.2 Long-lived particle scenarios sensitive to standard L1 triggers 6

3 Scenario with direct pair-production of LLPs and their decay to jets in the HL-LHC

3.1 Efficiency of standard Phase-II L1 triggers for LLPs 8

$\begin{array}{lll}3.2 & \text { Effect of high pile-up on jet distributions } & 12\end{array}$

4 Dedicated LLP triggers based on L1 tracking $\quad 16$

4.1 Training a BDT to classify displaced and prompt jets based on tracking $\begin{array}{ll}\text { information } & 18\end{array}$

4.2 Triggers based on the BDT training using variables from tracking 22

5 Performance of the classifiers based on tracking for some other LLP scenarios

5.1 Scenario with direct pair-production of LLPs and their decay to jets and invisible particle

5.2 Scenario where LLPs are produced from the decay of Higgs boson and decay to jets

5.3 Scenario where LLPs are produced from the decay of a heavy resonance and decay to jets

6 Conclusions and outlook

A Supplementary materials in developing the track-based L1 trigger

$\begin{array}{lll}\text { A.1 Distributions of tracking variables } & 35\end{array}$

$\begin{array}{lll}\text { A.2 Correlation matrix of variables } & 37\end{array}$

A.3 Trigger efficiencies and rates for $90 \%$ background rejection 39

A.4 The 200 PU scenario 40

\section{Introduction}

The RunII of LHC has ended in 2018 and no clear hint of new physics is found yet. LHC and its two general purpose detectors were mainly built to look for signatures of new physics involving prompt particles, which was widely searched for in LHC during both RunI and RunII. In the absence of any clear signal in that sector, the focus of LHC searches 
is shifting towards long-lived particles (LLPs) and in upcoming LHC runs LLP searches will be one of the priorities of the LHC experiments. The presence of a long-lived new particle can be a salient trait of several beyond standard model scenarios. A plethora of BSM models predict the presence of long-lived particles. Supersymmetry (SUSY) in different incarnations, several dark matter models, or portal models predicting interactions between a possible hidden sector and the SM are potential sources of LLP [1]. Among the SUSY models predicting LLPs [2-19], some of the most compelling ones are the R-parity violating SUSY, gauge and anomaly mediated SUSY breaking scenarios, split SUSY, and stealth SUSY. LLPs can also be present in many dark matter models [20-27]. In hidden valley scenarios [28-30], particles of the hidden sector such as long-lived dark photons or dark hadrons may decay to standard model particles. Axions or axion-like particles [31], which provide a nice resolution to the strong CP problem, can also have long lifetimes and can be probed in LHC in different final states.

Several LLP searches have been performed at the ATLAS and CMS experiments [3244], even though the detectors were a priori designed and optimised for promptly produced particles. For neutral LLPs that decay to charged particles, displaced vertex searches were carried out, while for charged LLPs, several unique signatures like disappearing track, kinked track, long time of flight in muon chamber, high ionisation energy loss per path length of a track $(\mathrm{dE} / \mathrm{dx})$ etc., were looked for. For a comprehensive review of various LLP models and experimental searches, the readers are referred to [45] and the references therein.

Depending on lifetime of LLP, the signature in detector can widely vary, thus inclusive searches are often not possible, and dedicated search strategies need to be designed. While LHC experiments have performed extensive searches to look for LLPs, there are some other interesting signatures that are yet to be searched for in the experiments, for example, difference in energy deposition patterns in the hadron calorimeter (HCAL) for displaced jets or backward moving objects originating from a heavy slow-moving or stopped LLP [46, 47] compared to the energy deposition pattern of prompt jets. Many of these signatures are experimentally challenging and usage of modern machine learning techniques would be beneficial to have better discovery potential.

When it comes to LLP search in LHC experiments, trigger poses a critical challenge. To overcome challenges of triggering LLP signatures, several novel ideas have been devised by the experimental collaborations. For example, the "CalRatio" trigger [48], which is a trigger which makes use of the ratio of energy deposited in HCAL and electromagnetic calorimeter (ECAL) for a particular jet. Another idea already used was to trigger on trackless jet signature [48] at high-level trigger, which provides a robust way to identify displaced jets coming from LLP that decayed after crossing the tracker. For LLP decays taking place just before or inside muon spectrometers, a special trigger, which looks for large number of charged hadrons traversing a narrow region of the muon spectrometer, was used by the ATLAS collaboration [48, 49].

In high-luminosity LHC (HL-LHC) [50] the triggering challenge will be more severe because of high pile up (PU). High PU makes it difficult to reconstruct the hard interaction, because a huge number of soft charged particles give rise to many tracks, which overlap 
spatially. Moreover, the calorimeter energy deposits can also overlap due to particles coming from many pp collisions. This can increase the rate of false triggers. It was found that the trackless jet and the CalRatio triggers are sensitive to the number of PU interactions and showed significant reduction in efficiency with increasing PU [48]. So it is understood that the efficiency and rate of the novel LLP triggers already used in experimental searches will be severely affected by high PU in HL-LHC, and in order to keep the rate under control the trigger level cuts might need to be tightened. However, it would be good to apply dedicated cuts rather than tighter cuts to keep the trigger rates in acceptable limits as well as to select LLP events efficiently. Since the cross sections of the LLP processes can be really small $\sim \mathcal{O}(\mathrm{fb})$ or even less, the goal must be to select as many LLP events as possible at the first level of the trigger system (level-1 or L1 for CMS). If the event does not pass the L1 trigger, we will lose it forever, and due to this the prospect of discovering LLPs at the LHC will be severely hampered.

In our paper, we will present the results in the context of CMS detector and its upgrade in HL-LHC. The improved detector systems of the CMS Phase-2 upgrades [51] will help in maintaining the necessary performance of object reconstruction and identification under the arduous conditions of high PU at the HL-LHC. A key component of the upgrade of CMS experiment for HL-LHC is a unique arrangement of the outer part of the tracker. Utilizing the strong magnetic field of the CMS detector, it will permit the use of tracks in the L1 trigger stage, with input event rate of $40 \mathrm{MHz}$, arising from pp bunch crossings [52]. This distinctive attribute, along with the opportunities rendered by advancement in FPGA processing capability and bandwidth, will permit more elegant L1 triggers. The new track trigger facility will be able to identify tracks with $p_{T}>2 \mathrm{GeV}$ at the hardware-based first-level trigger, L1. ${ }^{1}$

There are certain optimistic scenarios where PU won't be a major problem for LLP search and dedicated LLP triggers might not be essential. For example, when the LLP is very heavy and its hadronic decay products deposit enough energy in calorimeters to push the events pass the usual $H_{T}$ trigger thresholds, or when the LLP decays to muons and muon chambers being the last layer of the detector is least affected by PU, so the displaced muon signature will still be prominent even in high PU scenario. However, there are some pessimistic scenarios where LLP is not very heavy and its hadronic decay products are less energetic. The track-trigger upgrade of the CMS detector might be very useful for LLP searches, in situations where the LLP is light and decays to jets. In order to be sensitive to models where LLP decays to low $p_{T}$ jets, it is extremely important to have a good handle on PU mitigation. In this paper we have explored how the upgraded CMS detector might perform in the high PU environment of HL-LHC to differentiate between prompt and displaced jets. We design some dedicated LLP triggers for selecting LLP events efficiently keeping the background rates within the acceptable limit, using the L1 tracking.

The rest of the paper is outlined as: in section 2, we discuss the HL-LHC upgrades and L1 triggers in detail and the LLP scenarios that might not get selected with the standard

\footnotetext{
${ }^{1}$ Tracking displaced charged particles at L1 is challenging. There are some works going on to improve tracking at L1 to include LLP scenarios that will increase the sensitivity of LLP searches [53].
} 
triggers. In section 3, we define a particular LLP scenario, where LLPs are pair-produced directly and then decay to jets, and study the effect of PU on different distributions and also the performance of standard L1 jet triggers in selecting LLP events. In section 4, we develop some dedicated triggers for LLPs using the tracking information at L1, which can efficiently identify jets coming from the decay of light LLPs, unlike the standard jet triggers. In section 5, we briefly discuss a few other LLP scenarios, each highlighting a different aspect of triggering LLPs at L1. Finally, in section 6, we conclude.

\section{The high luminosity LHC and triggering long-lived particles}

In this section, we discuss the major upgrades for the high luminosity LHC and also some of the triggers at L1 of CMS relevant for this work. Although these triggers are optimized for standard prompt scenarios, we do not know how they perform for non-prompt cases. In the later part of this section, we briefly discuss which LLP scenarios will be easier to trigger using standard triggers or have some other sensitivity and which scenarios will be more difficult to trigger.

\subsection{The Phase-II HL-LHC upgrade and level-1 triggers}

We start with a brief discussion of the major upgrades for the high luminosity runs of the LHC. We will also discuss the standard L1 triggers for the HL-LHC runs. Peak instantaneous luminosity at HL-LHC will increase up to $\sim 7.5 \times 10^{34} \mathrm{~cm}^{-2} \mathrm{~s}^{-1}$ which is much higher than the current LHC design value of $1.0 \times 10^{34} \mathrm{~cm}^{-2} \mathrm{~s}^{-1}$ [50]. The increase in the instantaneous luminosity of the HL-LHC runs also mean an increase in the average PU rate, estimated to be close to $\langle\mathrm{PU}\rangle=140$ vertices for a peak luminosity of $\sim 5 \times 10^{34} \mathrm{~cm}^{-2} \mathrm{~s}^{-1}$ (and $\langle\mathrm{PU}\rangle=200$ for peak luminosity $\sim 7.5 \times 10^{34} \mathrm{~cm}^{-2} \mathrm{~s}^{-1}$ ) in each bunch crossing at the start (end) of the HL-LHC compared to the present number of $\langle\mathrm{PU}\rangle=30-50$ vertices per bunch crossing. To handle such a large amount of $\mathrm{PU}$ without compromising with its physics reach, the HL-LHC runs will witness many detector upgrades as proposed in the Phase-II upgrade of the detectors.

The Phase-II upgrade of the CMS tracker will include installation of a new silicon tracker (aka outer tracker) and a new pixel detector (aka inner tracker), which will have the capability to read out important information related to tracking, though limited, at $40 \mathrm{MHz}$ to the hardware-based L1 trigger system. It can read out the hits left only by high momentum tracks $\left(p_{T}>2 \mathrm{GeV}\right)$, out of the huge number of silicon hits, all of which cannot be read due to the latency limitation at L1. Inclusion of L1 tracking can help reduce the PU rate at the L1 triggers keeping the efficiency of interesting physical processes at par with those obtained for the present LHC runs. This will become clear when we discuss the standard L1 multijet triggers for CMS at HL-LHC. Tracker modules, made out of two sensors that are placed side by side, and an integrated circuit that harmonises the hits in them, provides both spatial and transverse momentum $\left(p_{T}\right)$ measurements. The pair of hits in such a module are called stubs. A track will produce a stub if hits in the sensors of a $p_{T}$ module fall in a specified window corresponding to a particular $p_{T}$ threshold. Tracks having $p_{T}$ below this threshold will be rejected. However, the $p_{T}$ measurement assumes 
that the tracks originate from the beamline, and therefore this will lead to wrong estimation of track parameters for displaced tracks coming from decays of long-lived particles, as has been discussed in [54].

Before discussing the HL-LHC L1 multijet triggers, let us briefly discuss how jets are usually formed at L1. In the current LHC runs, the L1 jets are formed using trigger towers, which are segmented as $\Delta \eta \times \Delta \phi$ size of $0.087 \times 0.087$ in the barrel (up to $|\eta| \sim 1.5$ ) and this size varies up to $\sim 0.17 \times 0.17$ as we move to $|\eta|=2.5$ (for the CMS detector) as given in the Delphes card for CMS. For the HL-LHC CMS upgrade, there is a proposition for High Granularity Calorimeters (HGCAL) at the endcaps of the detector, both for the electromagnetic and hadronic calorimeters which will have much smaller segmentations (with a size of $0.02 \times 0.02$ ) [55]. In this work, we do not use the HGCAL segmentation and instead use the uniform segmentation $(0.087 \times 0.087)$ for both barrel and endcaps up to $|\eta| \sim 3$ and then use the HF (hadronic forward calorimeter) segmentation. L1 Calo jets are usually clustered by taking all the towers up to $|\eta|=2.5$ which have transverse energy $\left(E_{T}\right)$ greater than $2 \mathrm{GeV}$. At L1, standard sequential jet formation algorithms like anti- $k_{T}[56]$ cannot be used due to limited time available for making trigger decisions. Therefore, jets are formed using sliding windows of size $9 \times 9$ around a tower with the maximum $E_{T}$, which has to be greater than $4 \mathrm{GeV}$. This reasonably matches with the anti- $k_{T}$ clustering with $R=0.4$ as shown in [57, figure 4], and takes much lesser time than the sequential clustering algorithms. We believe that in HL-LHC runs as well, the L1 jets formed with some particular sliding window size will resemble jets formed with anti- $k_{T}$ with the same cone size. Therefore, for this work, we use anti- $k_{T}$ algorithm to cluster jets.

Let us now discuss the L1 triggers designed for processes involving jets at the HL-LHC runs of CMS, and how they can control PU. The trigger menu involving more than one jet at the HL-LHC (dijet, quad jet, $H_{T}$ triggers, etc.) require all the jets contributing to the trigger selection to come from the same $z$-vertex. The same $z$-vertex condition is defined in terms of the L1 tracks associated with a jet. Tracks with $p_{T}>2 \mathrm{GeV}$, within $|\eta|<2.5$ having transverse displacement $\left(L_{x y}\right)$ less than $1 \mathrm{~cm}$ and within longitudinal spread of $\left|z_{0}\right|<30 \mathrm{~cm}$ are selected. The L1 tracks, satisfying above requirements, are associated with a $\mathrm{L} 1$ jet by taking all tracks within a cone of $\Delta R=0.4$ around the jet axis. The jet $z$-vertex is defined as the $p_{T}$ weighted average $z_{0}$ of all the tracks associated with the jet. The same $z$-vertex condition implies that the $z$-vertex of all jets that contribute to the trigger decision should satisfy, $\Delta z \leq 1 \mathrm{~cm}[51]$.

Based on this, the following jet triggers will be used at L1 of HL-LHC [51]:

- Single jet: at least one jet with $p_{T}>173 \mathrm{GeV}$; no tracking information needed. ${ }^{2}$

- Dijet: at least two jets with $p_{T}>136 \mathrm{GeV}$; both these jets should satisfy the same $z$-vertex condition.

- Quad jet: at least four jets with $p_{T}>72 \mathrm{GeV}$; all of these jets should satisfy the same $z$-vertex condition.

\footnotetext{
${ }^{2}$ The single jet trigger has a very high $p_{T}$ threshold and therefore, is not affected much by pile-up jets. Therefore, this trigger can be used even without the tracking information at L1.
} 
In addition to these triggers, there are the $H_{T}$ and MET triggers, which also use the tracking information. The $H_{T}$ trigger requires the total scalar $p_{T}$ sum of all the jets, $H_{T}>350 \mathrm{GeV}$. Also, all the jets contributing to $H_{T}$ should satisfy the same $z$-vertex condition. The MET trigger also requires all the objects (like electrons, muons and jets) which help the event pass the trigger come from the same vertex. All the above discussed thresholds are for 140 PU scenario. For the 200 PU case, these might be increased to keep the trigger rates within the allowed trigger bandwidth.

The same $z$-vertex condition added in the L1 triggers help in controlling the pile-up rate because pile-up jets are most likely to come from different $z$ positions. For instance, the dijet and quad jet trigger rates increase from 26 and $12 \mathrm{kHz}$ to 52 and $185 \mathrm{kHz}$ respectively, on removing the same $z$-vertex condition [58]. The same $z$-vertex condition, therefore, helps a lot to maintain the trigger rates for the above discussed thresholds, and as we can see the effect of PU is more in cases of triggers with low $p_{T}$ thresholds.

However, we don't know how this condition will affect triggering of events with LLPs, as these triggers are mostly optimized for prompt decays of particles. It is important to study the performance of standard L1 triggers for different LLP scenarios, to make sure we are not missing interesting events at the very first stage of the trigger system. Studying the performance of standard triggers can also guide us towards designing dedicated triggers for long-lived particles at L1. We want to address all these issues in this work by studying how efficiently can we select LLP events in the 140 PU environment of the HL-LHC with standard triggers and also how some dedicated LLP triggers might help at L1.

We will now present a brief discussion of various kinds of LLP models in the next section. We will also shed light on the scenarios where above mentioned standard trigger will be aptly efficient. In this work, we will mostly concentrate on LLP scenarios where we are not sure of the performance of the standard triggers.

\subsection{Long-lived particle scenarios sensitive to standard L1 triggers}

Long-lived particles can either be pair-produced directly or can come from the decay of a resonance. The standard L1 multijet triggers, as we have discussed in the previous section, will be able to trigger events with hard prompt particles, which can pass the $p_{T}$ thresholds as well as have enough associated tracks reconstructed at L1. The latter helps the final state objects to pass the same $z$-vertex condition. Therefore, if the LLP is produced along with prompt particles with high energies, it will get selected by the standard triggers. For instance, if the LLP is produced in the following process:

$$
p p \rightarrow A A, \quad A \rightarrow q \bar{q} X
$$

where $A$ decays promptly, $X$ is long-lived, and if the mass difference between $A$ and $X$ is large, then there will be hard prompt jets in the events which can help trigger such events. For similar reasons, events where LLPs come from the decay of the Higgs boson when the latter is produced through vector boson fusion (VBF) are easy to trigger as shown in [59].

Scenarios where the LLPs are pair-produced without any other prompt particles, like

$$
p p \rightarrow A, \quad A \rightarrow X X, \quad \text { or } \quad p p \rightarrow X X
$$


might suffer due to non-fulfillment of the same $z$-vertex condition. Since the L1 tracks can be reconstructed with high efficiency up to a transverse distance of only $1 \mathrm{~cm}$ from the beamline $\left(L_{x y}<1 \mathrm{~cm}\right)$ [52], if the LLPs decay after that, their decay products mostly won't have any associated tracks and therefore, won't be able to satisfy the same $z$-vertex condition.

However, if the LLP is really massive and decays to quarks or gluons, its decay products will have very high transverse momenta, which can be easily triggered by standard single jet trigger which does not use the L1 tracking information. Say, we have events where LLPs of mass $500 \mathrm{GeV}$ are pair produced and they decay to jets, then at least one of these jets will have $p_{T}>173 \mathrm{GeV}$ and pass the single jet trigger. ${ }^{3}$

The decay mode of the LLP is also an important factor in its searches. If the LLP decays to final states involving electrons, muons or photons, the final states are cleaner and also easier to trigger than if the LLP decays to quarks (or gluons) which hadronise to form jets.

Based on discussions till now, we find that LLP scenarios in increasing order of the difficulty to trigger them at L1 are:

- LLPs with associated hard and prompt particles,

- LLPs without associated hard and prompt particles

- massive LLPs decaying to jets,

- LLPs decaying to electrons, photons, muons

- LLPs with moderate masses and moderate decay lengths decaying to jets

- very light LLPs decaying to jets

where by moderate LLP masses, we mean cases where the decay products don't have enough $p_{T}$ to pass the trigger threshold, and by moderate decay lengths, we mean that significant amount of decays happening within the tracker volume. By very light LLPs, we mean a ballpark of a few $\mathrm{GeV}$, which can never lead to significant energy deposition in the calorimeters and therefore, will mostly require associated hard and prompt particles to pass the L1 trigger. They can also be triggered if they are produced with a hard ISR jet, but this will again reduce the cross-section of the process (we discuss this later in section 5).

In this work, we concentrate on the second most difficult possibility where the LLPs are not too heavy (around 10-100 GeV), they are produced without any hard prompt particles, their decay length is moderate such that they mostly decay within the tracker and they decay into final states with jets with $100 \%$ branching. We discuss four scenarios based on this - the first two with direct pair-production of LLPs and the other two where the LLP comes from the decay of the SM Higgs boson and a heavy resonance respectively. The first two scenarios differ in the decay mode of the LLP - one where the LLP decays to

\footnotetext{
${ }^{3}$ If the LLP decays mostly after the ECAL and before the HCAL, one can use the CalRatio trigger for selecting such events, where the ratio of the ECAL to HCAL energy deposition is low. Such triggers have been proposed by the ATLAS collaboration [48,49], and they will be mostly sensitive to LLPs with high decay length. However, the performance of such a trigger in the high PU environment of HL-LHC is yet to be studied, and therefore, we do not discuss this further in the present work.
} 
jets only, and the second where it decays to jets and an invisible particle. The motivation for the latter decay mode is to discuss the possibility of using a missing transverse energy trigger for such LLPs. We present our analyses with some benchmark points from the first scenario defined in the next section and discuss briefly the other scenarios in section 5 .

\section{Scenario with direct pair-production of LLPs and their decay to jets in the HL-LHC}

In this section, we calculate the signal efficiencies for some benchmarks from the scenario, where LLPs are pair-produced directly and then decay to jets, using the single jet, dijet and quad jet triggers. Later, we also discuss how adding $140 \mathrm{PU}$ affects the jet distributions in both LLP as well as background events. In this work, we study only the dominant background - QCD dijet events.

\subsection{Efficiency of standard Phase-II L1 triggers for LLPs}

In this section, we define our first LLP scenario (A), discuss the fractions of decays that we expect in different parts of the detector for various benchmark points (corresponding to different LLP masses and $c \tau$ values) in this scenario and also quote their L1 trigger efficiency when the LLP decays into jets and when the standard jet triggers are used at the HL-LHC.

We start with the following LLP scenario, which is just the direct pair-production of LLPs. Later in section 5, we present a discussion about some other LLP scenarios.

$$
\text { (A) } \mathbf{p p} \rightarrow \mathbf{X X}, \quad \mathbf{X} \rightarrow \mathbf{j j}
$$

We generate, using PYTHIA6[60], LLPs from a quark initiated $Z$-mediated process, ${ }^{4}$ where they are directly produced and then decay to two quarks, which hadronise to give jets. We study three different mass points - $50 \mathrm{GeV}, 100 \mathrm{GeV}$, and $200 \mathrm{GeV}$ having mean proper decay lengths ${ }^{5}$ of $10 \mathrm{~cm}$ and $100 \mathrm{~cm}$ each. The LLP is assumed to decay to light jets with $100 \%$ branching. We denote each benchmark point as "( $\left.\mathrm{M}_{-}, c \tau_{-}\right)$" with the mass of the LLP in $\mathrm{GeV}$ and the decay length $c \tau$ in $\mathrm{cm}$.

Jets are defined as clusters of ECAL and HCAL energy deposition towers using anti- $k_{T}$ jet clustering algorithm with $R=0.4$. We use Delphes-3.4.1[61] for detector simulation in our work with some modification such that the displaced stable particles deposit energy in the $\eta$ and $\phi$ bins corresponding to the detector segmentation rather than their actual $\eta-\phi$. LLPs decaying beyond the radial and half-length values from where the HCAL starts won't have any energy deposits in our setup. ${ }^{6}$ Before studying the trigger efficiency of these

\footnotetext{
${ }^{4}$ We use a supersymmetric LQD-type R-parity violating coupling, where a pair of sneutrinos are directly produced and they decay to two quarks, to simulate this type of LLP scenario.

${ }^{5}$ Hereafter, we will mostly refer to the mean proper decay length as just decay length, unless stated otherwise.

${ }^{6}$ In experiment, the HCAL has longitudinal segmentation, and therefore, still there will be jets formed even if the LLP decays after the HCAL starts, until the LLP decays within the HCAL. In Delphes however, there is no longitudinal segmentation and the energy of all hadrons are deposited where the HCAL starts. Therefore, we won't get jets once the decay happens after this radial and half-length value.
} 
benchmarks, we need to quantify the amount of decays occurring in various detector parts. This fraction depends on the boost (which is related to the mass and the production mode, whether direct or from a resonance) and the proper lifetime of the LLP as well as the volume of each detector part. The decay length of the LLP in the lab frame, $d$, is related to the boost of the LLP, $\beta \gamma$, and mean lifetime of the LLP in its rest frame, $\tau$, as

$$
d=\beta \gamma c \tau
$$

where $c$ is the velocity of light. We divide the decays into the following exclusive regions, where we have followed the CMS detector geometry [62]:

- Reco as L1 tracks: corresponds to transverse distance $\left(L_{x y}\right)$ of $1 \mathrm{~cm}$ from the beamline and half-length $\left(\left|z_{0}\right|\right)$ of $30 \mathrm{~cm}$; if the LLP decays within this region, the tracks of its decay products are expected to be reconstructed at L1, typically with $\sim 95 \%$ efficiency and hence it stands a chance to pass the L1 trigger with the same $z$-vertex condition.

- Before MTD: ${ }^{7}$ corresponds to transverse distance of $1.161 \mathrm{~m}$ and half-length of $2.6 \mathrm{~m}$; for this fraction of LLP events, the decay products of may not have L1 reconstructed tracks.

- Before ECAL: corresponds to transverse distance of $1.29 \mathrm{~m}$ and half-length of $3 \mathrm{~m}$; jets from the LLPs decaying in this region won't have many tracks reconstructed at L1.

- Before HCAL: corresponds to transverse distance of $1.811 \mathrm{~m}$ and half-length of $3.9 \mathrm{~m}$; jets from the LLPs decaying in this region won't have tracks reconstructed at L1. ${ }^{9}$

- Before MS: decays before the muon spectrometer (MS); corresponds to transverse distance of $4.020 \mathrm{~m}$ and half-length of $5.68 \mathrm{~m}$; from here on, we won't have any calorimeter energy deposit and hence, no jets.

- Inside MS: decays inside the muon spectrometer (MS); corresponds to transverse distance of $7.38 \mathrm{~m}$ and half-length of $10.86 \mathrm{~m}$.

- Outside detector: the LLP decays outside the detector.

Table 1 shows the percentage of the LLP decays in different detector parts for different benchmarks from scenario (A). From table 1, we notice a general trend that for a particular production mode of the LLP, with decreasing mass (increasing boost) and increasing proper decay length, the decay length in the lab frame shifts to higher values, as is expected from eq. (3.1). Therefore, we get relatively higher fraction of decays in the later parts of the detector. However, the $50 \mathrm{GeV}$ point in this scenario is different because at LLP mass values

\footnotetext{
${ }^{7}$ The MIP Timing Detector (MTD) is proposed to be included in the Phase-II upgrade of CMS between the Tracker and ECAL [63]. ${ }^{8}$

${ }^{8}$ This document describes a MIP timing detector for the Phase-2 upgrade of the CMS experiment, in view of HL-LHC running.

${ }^{9}$ In Delphes, the electrons and photons deposit energy only in the ECAL, therefore, jets after the ECAL won't have energy contribution from photons and electrons in our setup.
} 


\begin{tabular}{|c|c|c|c|c|c|c|c|}
\hline $\begin{array}{c}\text { Mass }[\mathrm{GeV}], \\
\text { Decay Length }[\mathrm{cm}]\end{array}$ & $\begin{array}{c}\text { Reco as } \\
\text { L1 tracks }\end{array}$ & $\begin{array}{c}\text { Before } \\
\text { MTD }\end{array}$ & $\begin{array}{c}\text { Before } \\
\text { ECAL }\end{array}$ & $\begin{array}{c}\text { Before } \\
\text { HCAL }\end{array}$ & $\begin{array}{c}\text { Before } \\
\text { MS }\end{array}$ & $\begin{array}{c}\text { Inside } \\
\text { MS }\end{array}$ & $\begin{array}{c}\text { Outside } \\
\text { detector }\end{array}$ \\
\hline 50,10 & 13.20 & 80.51 & 1.26 & 1.85 & 1.72 & 1.19 & 0.26 \\
50,100 & 1.38 & 46.55 & 3.38 & 7.58 & 11.02 & 12.55 & 17.55 \\
100,10 & 10.59 & 86.81 & 0.70 & 0.91 & 0.68 & 0.28 & 0.02 \\
100,100 & 1.15 & 49.46 & 3.71 & 8.84 & 13.66 & 12.31 & 10.88 \\
200,10 & 10.83 & 88.42 & 0.27 & 0.31 & 0.14 & 0.02 & 0.002 \\
200,100 & 1.12 & 56.27 & 3.79 & 9.66 & 14.27 & 9.72 & 5.17 \\
500,10 & 11.94 & 87.99 & 0.04 & 0.04 & 0.04 & 0 & 0 \\
500,100 & 1.32 & 66.65 & 3.75 & 9.54 & 12.80 & 4.86 & 1.10 \\
\hline
\end{tabular}

Table 1. Percentage of decays for different benchmark points from scenario (A) in various detector parts using the CMS detector dimensions.

of $40-50 \mathrm{GeV}$, they will preferentially be produced by the decay of an on-shell $Z$-boson, since it is allowed in the way we are generating the LLPs through a $Z$ mediated process, and hence they will have very little boost and smaller decay lengths. This dependence of the fraction of decays of the LLP, in various regions of the tracker, on its mass and $c \tau$ is also illustrated in [64].

Figure 1 shows the multiplicity (left) and $p_{T}$ (right) distribution of jets with transverse momentum greater than $15 \mathrm{GeV}$ from decays of LLPs from scenario (A) characterized by different masses and decay lengths. The jet multiplicity increases with mass of the LLP and decreases with increasing decay length, as is expected since massive LLPs and LLPs with smaller decay lengths decay more often within the starting of the HCAL (see table 1). For highly displaced LLP decays, like when the LLP decays just before the HCAL, the two jets from the decay can be close enough to get identified as a single jet, and this also affects the multiplicity.

The jet $p_{T}$ distribution becomes harder with increasing mass of the LLP. With increasing decay length, the $p_{T}$ distribution becomes slightly softer due to the fact that higher $p_{T}$ jets will most probably come from LLPs with larger boosts, and these will mostly decay after the HCAL starts, and hence are not identified as jets.

We now move towards finding the trigger efficiency of our LLP benchmarks when standard jet triggers are used at L1. To find trigger efficiency, we need to merge our LLP samples with pile-up. We generate 1 million soft QCD events using PYTHIA8[65] and use this as the pile-up. We merge the PU with the hard process using the PileUpMerger ${ }^{10}$ of Delphes-3.4.1 with each event having $\langle\mathrm{PU}\rangle=140(200)$ vertices in addition to a hard $p p$ collision vertex. Figure 2 shows the spread of PU vertices in 10,000 $p p \rightarrow \nu \nu$ events merged with $\langle\mathrm{PU}\rangle=140$ vertices per bunch crossing. The number of $\mathrm{PU}$ events merged with each

\footnotetext{
${ }^{10}$ We had to modify the PileUpMerger code of Delphes slightly as it was cancelling offset in the $z$ and $t$ values with respect to the first stable particle, which will clearly be a problem when our first stable particle comes from the decay of the LLP.
} 

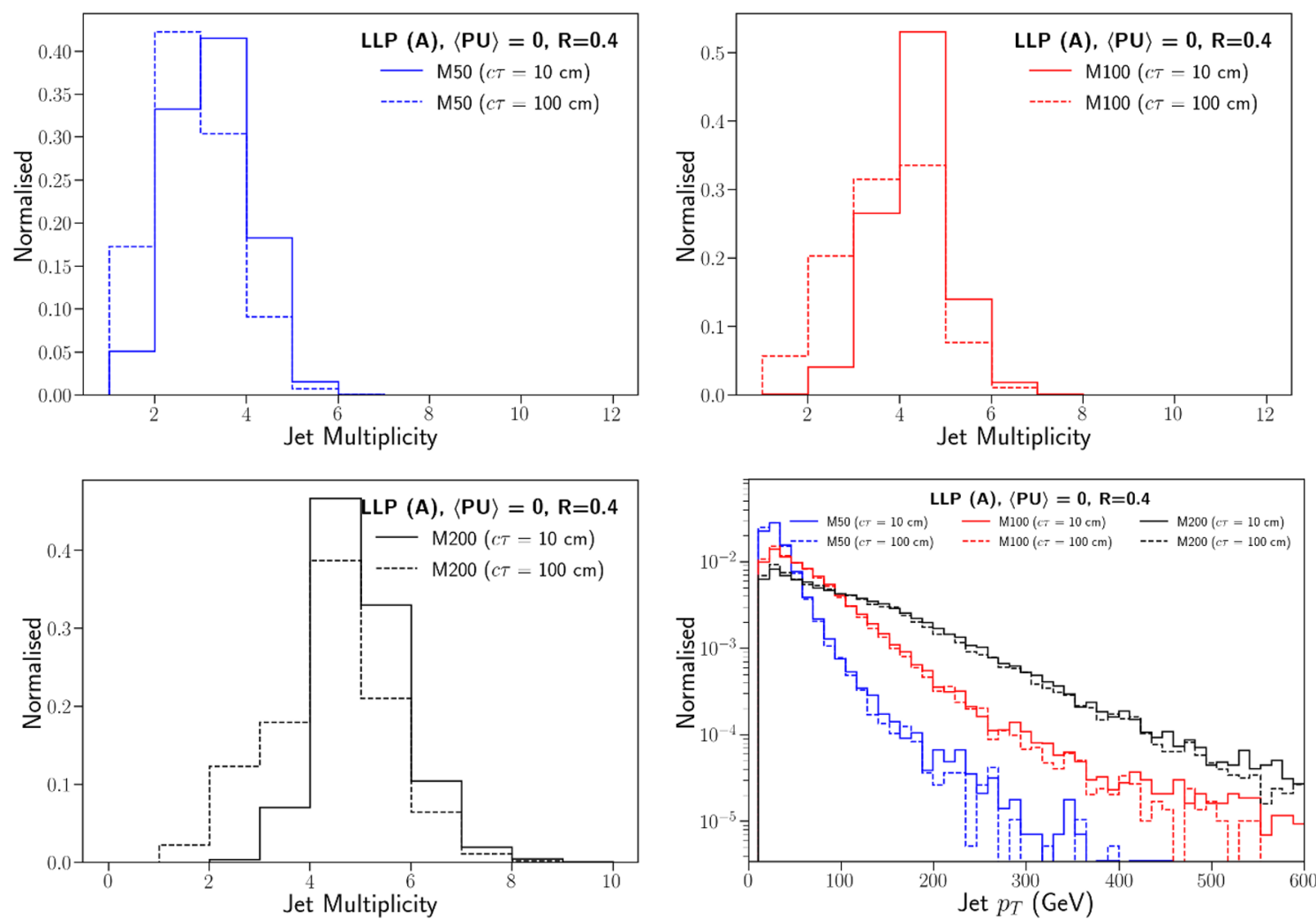

Figure 1. Multiplicity (top panel and bottom left) and $p_{T}$ (bottom right) distribution of jets with $p_{T}>15 \mathrm{GeV}$ coming from the decay of LLPs with different masses and decay lengths from scenario (A) as described above. The distributions are normalised such that their area is 1 .

hard collision follows a Poisson distribution with average value of 140 . The details of how the PU vertices are spread in the $z$ and time directions are described in the caption of figure 2.

Table 2 shows the signal efficiencies when standard single jet, dijet and quad jet triggers are used on LLP events from benchmark points in scenario (A). We present the efficiencies before (0 PU) and after merging with 140 PU. For comparison, we also present the dijet and quad jet efficiencies without the same $z$-vertex condition, which does not need the L1 track information (columns without "(trk)"). Adding PU increases the trigger efficiency for the signal benchmarks, as we can see from table 2. The trigger efficiencies for the quad jet (trk) trigger is increased from $\sim 0$ to $\sim 10 \%$ in the presence of PU for the $50 \mathrm{GeV}$ benchmark point. Energy depositions coming from PU might increase the $p_{T}$ of the LLP jets. However, even if they satisfy the $p_{T}$ threshold, these jets will have very less probabilities of having tracks reconstructed at L1 (see table 1), and therefore, they won't satisfy the same $z$-vertex condition. Jets from PU processes, on the other hand, can satisfy the $p_{T}$ threshold as well as the same $z$-vertex condition together in some cases and therefore, help trigger some of the LLP events. One way to see that these events are indeed triggered due to the PU jets is the fact that quad jet (trk) trigger efficiencies don't depend as much on the decay length of the LLP as their percentage of decaying within the region where the L1 tracks can be 


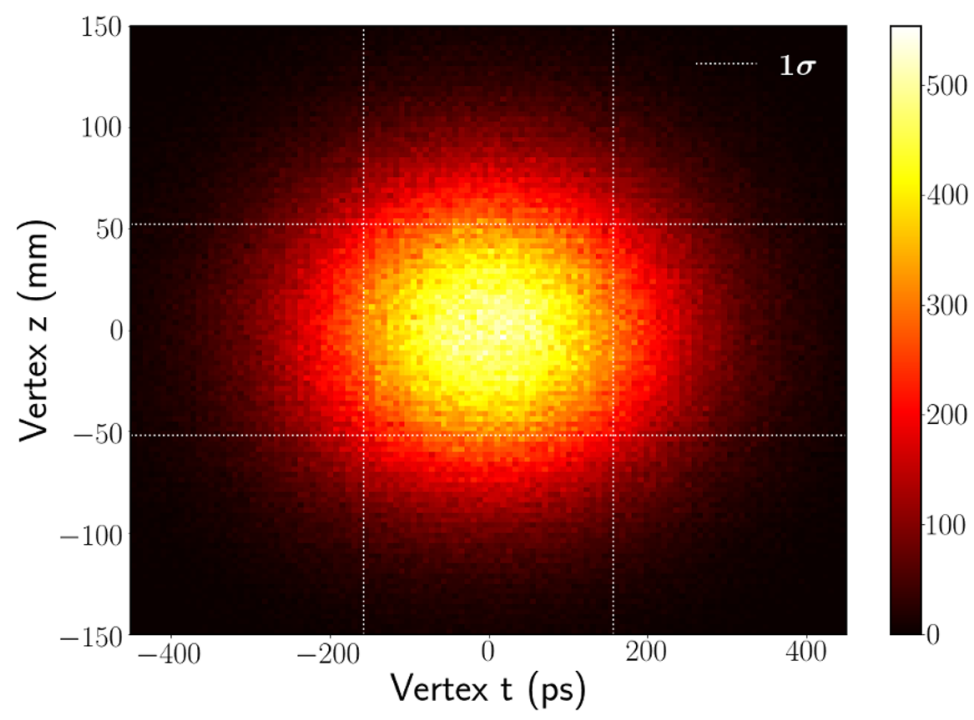

Figure 2. The spread of $z$ positions and timings of 140 average PU vertices for 10,000 events. The color bar indicates the number of vertices having a particular $z$ position and time $t$. The vertices follow a two-dimensional Gaussian distribution, with $z=0$ and $t=0$ having the maximum probability of having a vertex. The total spread is of $25 \mathrm{~cm}$ and $800 \mathrm{ps}$ respectively in $z$ and $t$ directions. The dotted grey lines correspond to the $1 \sigma$ values of the Gaussian distributions in the $z$ and $t$ directions, with $\sigma_{z}=5.3 \mathrm{~cm}$ and $\sigma_{t}=160 \mathrm{ps}$ respectively.

efficiently reconstructed depends (see the second column of table 1). Also, the effect of PU is dominant for the quad jet triggers than the single and double jet ones due to the lower $p_{T}$ threshold of the former.

We also find that removing the same vertex condition is helpful in improving the trigger efficiency, but it again comes with the cost of increasing the rates to very high values because of high PU and decreasing the purity of the LLP sample. As we have discussed earlier in section 2.1, the quad jet rate increases from $12 \mathrm{kHz}$ to $185 \mathrm{kHz}$, on lifting up the same $z$-vertex condition. Since we are limited by the trigger bandwidth, we need additional conditions after lifting up the same vertex condition to control the trigger rate. These conditions should be such that they are able to remove most of the PU contribution and can then differentiate between hard processes involving long-lived particles and QCD ones.

To achieve this, we first need to study how pile-up affects the jet distributions for the LLP events, which is our signal, and QCD dijet events, which is the dominant background.

\subsection{Effect of high pile-up on jet distributions}

The same $z$-vertex condition, though is a great way to control PU rates, it affects the signal efficiency of LLP events adversely as we have found in the previous section. However, if we want to lift up this condition, we have to study how the pile-up contribution affects the signal and background distributions. As discussed in the previous section, we will study the dominant background coming from QCD dijet events in this work. We generate the QCD dijet events with different exclusive $p_{T}^{\text {gen }}$ cuts at the generation level of the partons $-p_{T}^{\text {gen }} \in(50,100) \mathrm{GeV}, p_{T}^{\text {gen }} \in(100,150) \mathrm{GeV}, p_{T}^{\text {gen }} \in(150,200) \mathrm{GeV}$ and $p_{T}^{\text {gen }}>200 \mathrm{GeV}$, 


\begin{tabular}{|c|c|c|c|c|c|c|}
\hline $\begin{array}{c}\text { Number of } \\
\langle\mathrm{PU}\rangle\end{array}$ & $\begin{array}{c}\text { Mass [GeV], } \\
\text { Decay Length }[\mathrm{cm}]\end{array}$ & $\begin{array}{c}\text { Single } \\
\text { jet }\end{array}$ & Dijet & $\begin{array}{c}\text { Dijet } \\
(\text { trk })\end{array}$ & $\begin{array}{c}\text { Quad } \\
\text { jet }\end{array}$ & $\begin{array}{c}\text { Quad } \\
\text { jet (trk) }\end{array}$ \\
\hline \multirow{3}{*}{$\langle\mathrm{PU}\rangle=0$} & 50,10 & $0.92 \%$ & $0.83 \%$ & $0.03 \%$ & $0.01 \%$ & $0.00 \%$ \\
& 50,100 & $0.50 \%$ & $0.17 \%$ & $0.02 \%$ & $0.00 \%$ & $0.00 \%$ \\
& 100,10 & $11.39 \%$ & $10.20 \%$ & $0.83 \%$ & $1.24 \%$ & $0.05 \%$ \\
& 100,100 & $8.26 \%$ & $3.44 \%$ & $0.53 \%$ & $0.18 \%$ & $0.03 \%$ \\
& 200,10 & $54.40 \%$ & $47.73 \%$ & $7.08 \%$ & $13.40 \%$ & $0.67 \%$ \\
& 200,100 & $43.73 \%$ & $25.72 \%$ & $4.36 \%$ & $3.99 \%$ & $0.28 \%$ \\
\hline PU $\rangle=140$ & 50,10 & $2.72 \%$ & $2.81 \%$ & $0.47 \%$ & $59.65 \%$ & $10.99 \%$ \\
& 50,100 & $1.78 \%$ & $0.94 \%$ & $0.25 \%$ & $55.94 \%$ & $10.57 \%$ \\
& 100,10 & $27.43 \%$ & $28.03 \%$ & $5.83 \%$ & $75.85 \%$ & $14.21 \%$ \\
& 100,100 & $20.48 \%$ & $12.59 \%$ & $2.93 \%$ & $66.94 \%$ & $12.26 \%$ \\
& 200,10 & $81.04 \%$ & $77.71 \%$ & $23.68 \%$ & $89.19 \%$ & $18.23 \%$ \\
& 200,100 & $67.98 \%$ & $50.11 \%$ & $13.98 \%$ & $77.18 \%$ & $14.86 \%$ \\
\hline
\end{tabular}

Table 2. Efficiency of selecting LLP events for benchmark points from scenario (A) with the standard Phase-II L1 jet triggers both for zero PU and 140 PU scenarios. For the multijet triggers, efficiencies for both, without and with (columns with '(trk)') the same- $z$-vertex condition are quoted.

using PYTHIA6. Merging the hard processes with PU affects the jet multiplicity as well as other distributions, and since we have around $\langle\mathrm{PU}\rangle=140$ vertices per bunch crossing, $\mathrm{PU}$ contribution will be dominant. We start by looking at the jet multiplicity distribution for the signal and the background with different average $\mathrm{PU}(\langle\mathrm{PU}\rangle)$ values.

We have to study jets having transverse momentum above some threshold, and if this is very low, it will be significantly affected by the pile-up, and if this is too high, our signal jets from lighter LLPs won't be selected. Since our background QCD dijet events have been generated starting from a $p_{T}^{\text {gen }}$ cut of $50 \mathrm{GeV}$, we consider jets with a transverse momentum greater than $60 \mathrm{GeV}$. Figure 3 shows the number of jets having $p_{T}>60 \mathrm{GeV}$ and $|\eta|<2.5$ coming from signal (a LLP benchmark point from scenario (A)) and background (QCD with $\left.p_{T}^{\text {gen }} \in(100,150) \mathrm{GeV}\right)$ with $0 \mathrm{PU}, 140 \mathrm{PU}$ and $200 \mathrm{PU}$. We find that increasing the amount of PU shifts the average number of jets from 3-4 (for 0 PU) to about $\sim 10$ and $\sim 40$ for 140 and 200 PU scenarios respectively, for both the LLP benchmark point and QCD dijet events.

Clustering jets with $R=0.4$ on the $140 \mathrm{PU}$ (200 PU) sample, therefore, significantly affects the distributions coming from the sample with no PU. Also, this bias is the same for both QCD and LLP processes and it will make both of their distributions look alike, even if they were different to start with, since the PU jets dominate both the samples. Therefore, it becomes hard to differentiate between them.

Therefore, if we want to lift up the same $z$-vertex condition for improving the LLP signal efficiencies, we have to use some other ways for reducing the pile-up contribution. The 


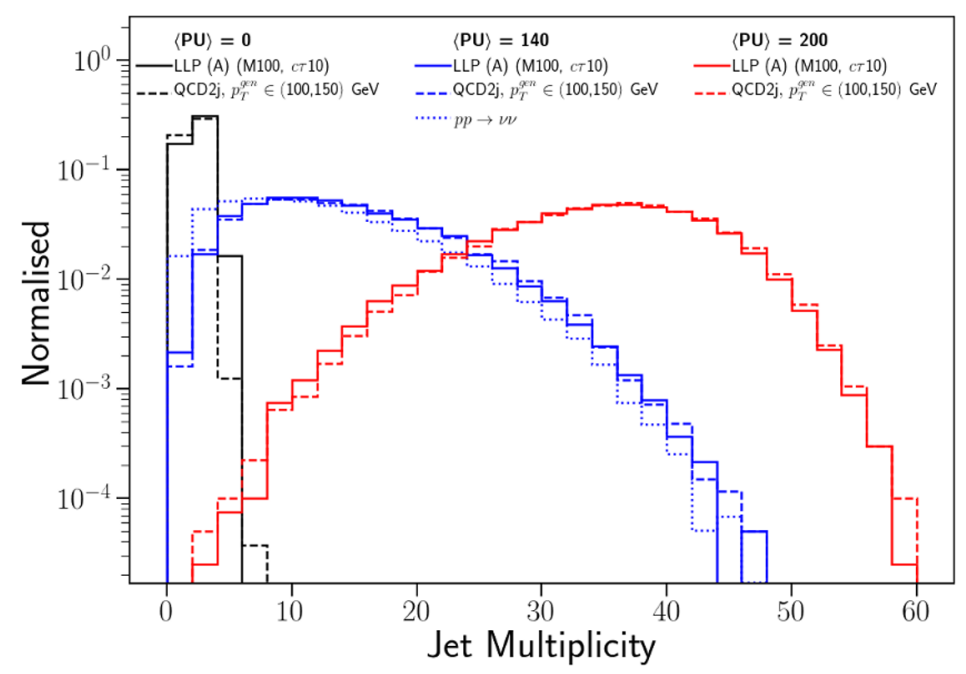

Figure 3. Number of jets coming from QCD dijet processes (background) and LLP process corresponding to scenario (A), with $M_{X}=100 \mathrm{GeV}$ and $c \tau=10 \mathrm{~cm}$ (signal) with zero PU, $140 \mathrm{PU}$, and 200 PU. Number of jets coming from a process having just 140 PU (generated by merging 140 PU with $p p \rightarrow Z \rightarrow \nu \nu$ process) is also shown.

pile-up contribution is mostly uniform throughout the detector and therefore, its effect will depend on the area of the jet. Narrow jets with less area in the $\eta \times \phi$ plane will, therefore, have smaller PU contribution and also, it is more unlikely for a PU jet to contain more than $60 \mathrm{GeV}$ transverse momentum within a smaller region of $\eta \times \phi$. Therefore, we can reduce the multiplicity of jets coming from the PU collisions.

The cone size of hadronic objects need to be motivated by the physical size of the spread of the object in $\eta-\phi$. The cone size should be such that it is big enough to fully or at least mostly contain the hadronic activity of the jets from signal events, and small enough to not include a huge amount of PU contribution, while it is understood that some PU contamination would be unavoidable. If most of the energy of the signal jets is not contained within the smaller cone size, they won't be able to satisfy the $p_{T}$ threshold, and it will decrease the signal efficiency. For jets coming from the decay of long-lived particles, we have another advantage of considering narrow jets. Displaced jets from LLP decays will have energy deposition contained in smaller region as has been pointed out in [36] and also discussed in [47]. Therefore, their $p_{T}$ might be affected less if we consider narrow jet cone sizes.

We compare the jet $p_{T}$ distributions with different cone sizes $(R=0.1,0.2,0.3,0.4)$, for one benchmark point of signal from scenario (A), (M100, $c \tau 10)$ (left) and for the background QCD dijet events with $p_{T}^{\text {gen }} \in(50,100) \mathrm{GeV}$ (right) without PU in figure 4 for all jets with $p_{T}>60 \mathrm{GeV}$ and $|\eta|<2.5$ cuts in 10,000 events. We find that the $p_{T}$ distribution becomes softer as is expected with reducing cone size $R$. We find that the QCD jets with $p_{T}^{\text {gen }} \in(50,100) \mathrm{GeV}$ are affected much more than the LLP benchmark, which is expected since they start from the beamline and spread more in the physical region till they reach the calorimeters and deposit energy in a larger cone of radius $R=0.4$ mostly. As the $p_{T}$ 

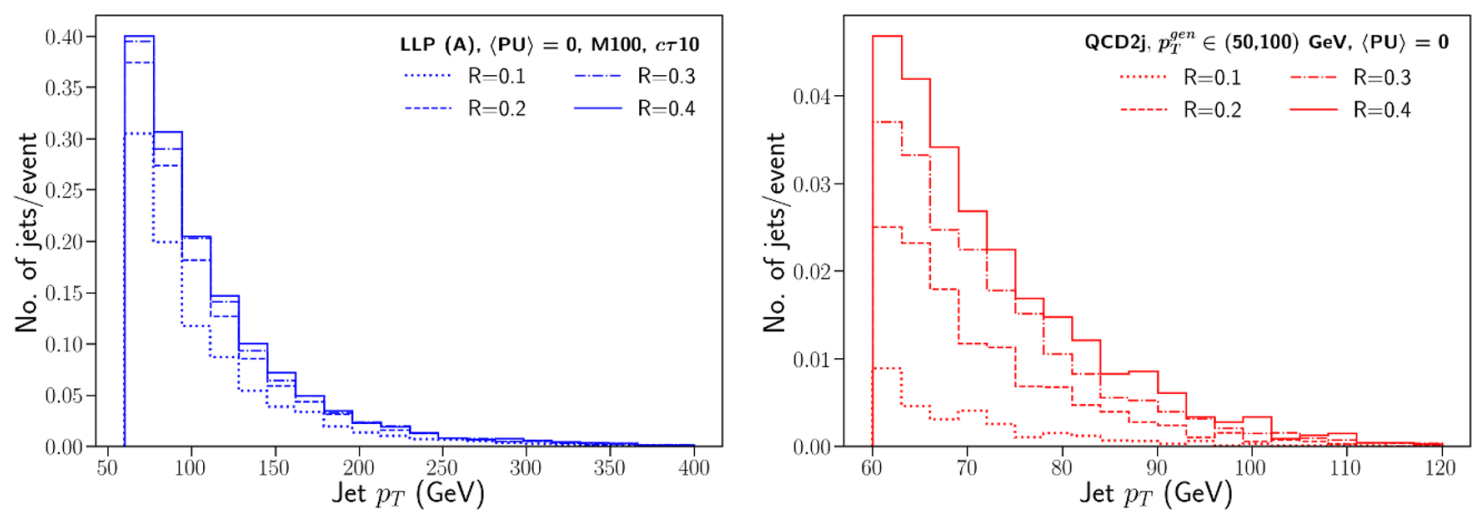

Figure 4. Comparison of jet $p_{T}$ distributions for jets clustered using anti- $k_{T}$ with cone sizes $R=0.1, R=0.2, R=0.3$ and $R=0.4$ coming from LLPs with mass of the LLP $100 \mathrm{GeV}$ and decay length $10 \mathrm{~cm}$ in scenario $(\mathrm{A})$ (left) and from QCD dijet events with $p_{T}^{\text {gen }} \in(50,100) \mathrm{GeV}$ without any PU. The distributions are drawn for 10,000 events.
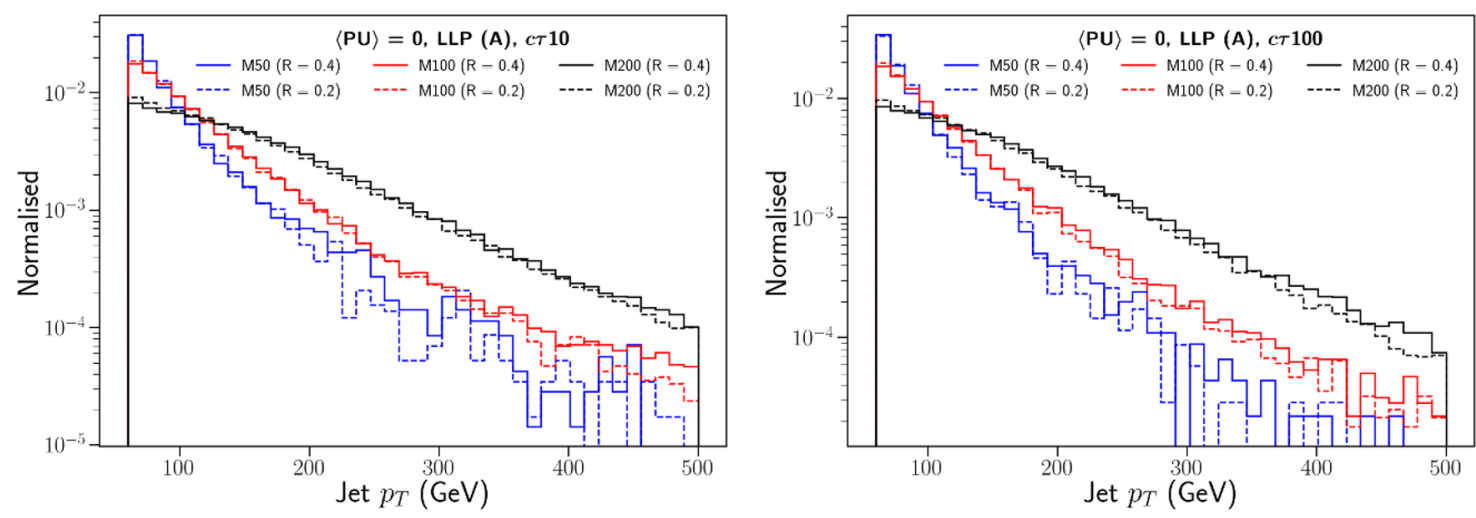

Figure 5. Comparison of jet $p_{T}$ distributions for jets clustered using anti- $k_{T}$ with $R=0.2$ and $R=0.4$ coming from LLP benchmark points from scenario (A) with different masses having a decay length of $10 \mathrm{~cm}$ (left) and $100 \mathrm{~cm}$ (right) with zero PU.

of the QCD jet increases, the jet constituents will be more collimated and hence, narrow jets won't affect the $p_{T}$ distribution much.

Our finding is also supported by experimental collaborations, which have used small cone size when it is known that the hadronic object of interest is expected to be narrow. For example, ATLAS collaboration have used $R=0.2$ cone size for tau jet triggers [66], as hadronic taus are narrower than their QCD counterparts. We decide to use $R=0.2$ jets because the signal $p_{T}$ distribution is affected less till that cone size value. Figure 5 compares the jet $p_{T}$ distribution for the LLP benchmark points from scenario (A) with decay length $10 \mathrm{~cm}$ (left) and $100 \mathrm{~cm}$ (right) using the usual $R=0.4$ jets with narrow jets $(R=0.2)$. We find that using narrow jets does not change the jet $p_{T}$ distribution for the LLP hard process much.

Let us now add PU and see how considering narrow jets help. The left panel of figure 6 shows the comparison of the jet multiplicity distribution coming from a LLP benchmark 

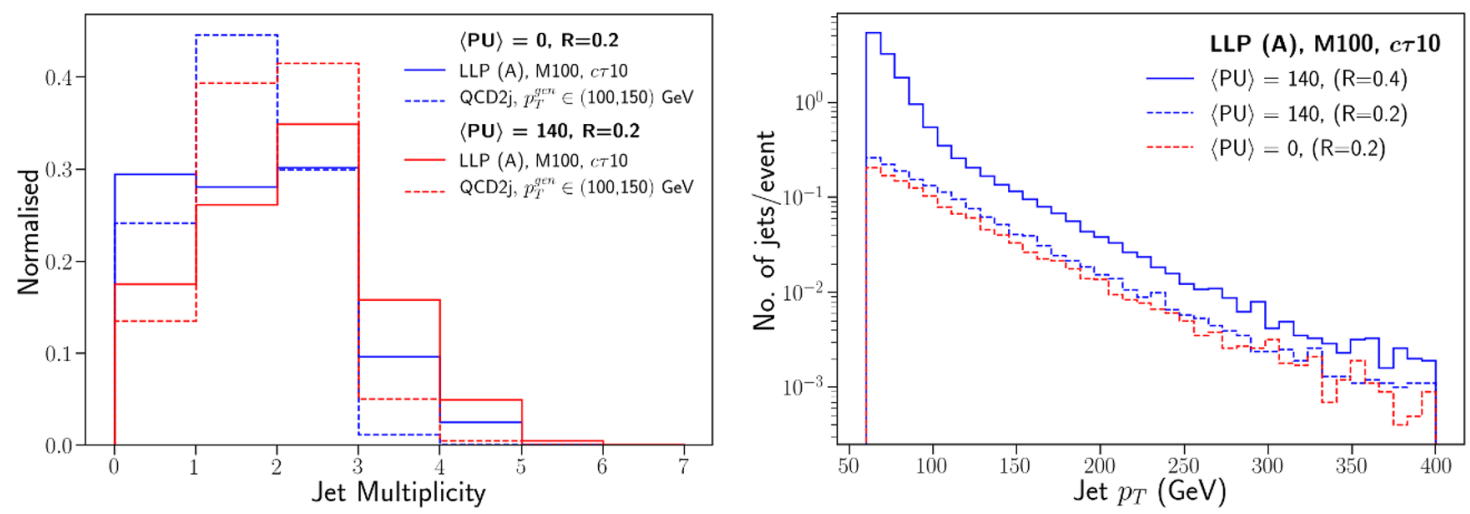

Figure 6. Left: jet multiplicity for jets clustered with anti- $k_{T} R=0.2$ having $p_{T}>60 \mathrm{GeV}$ from LLP benchmark (M100, $c \tau 10)$ from scenario (A) and QCD dijet events with $p_{T}^{\text {gen }} \in(100,150) \mathrm{GeV}$ merged with no PU and $140 \mathrm{PU}$; Right: $p_{T}$ distribution for jets clustered using anti- $k_{T}$ with $R=0.2$ and $R=0.4$ coming from LLP benchmark $\left(M_{X}=100 \mathrm{GeV}\right.$ and $\left.c \tau=10 \mathrm{~cm}\right)$ from scenario (A) with $140 \mathrm{PU}$ compared with the zero PU jet $p_{T}$ distribution, all drawn for 10,000 events.

point from scenario (A) and from QCD dijet events with 0 PU and 140 PU clustered with $R=0.2$, and the right panel of figure 6 shows the comparison of the $p_{T}$ distribution of jets coming from a LLP benchmark point from scenario (A) with 0 PU (with $R=0.2$ ) and with $140 \mathrm{PU}$ for jets with $R=0.2$ and $R=0.4$. A similar figure with $200 \mathrm{PU}$ events is shown in figure 22 in appendix A.4. We find that the multiplicity of jets with $p_{T}>60 \mathrm{GeV}$ decreases when we consider $R=0.2$ jets from that when we had $R=0.4$ jets. The multiplicity distribution does not exactly match the zero PU multiplicities, but we are able to get rid of many PU jets. The $p_{T}$ distribution is for all the jets with $p_{T}>60 \mathrm{GeV}$ and $|\eta|<2.5$ in 10,000 events for each case. When jets are clustered using anti- $k_{T}$ with $R=0.4$, processes merged with $140 \mathrm{PU}$ have very high jet multiplicities (figure 3), most of which are PU jets with low $p_{T}$ values. Therefore, the $p_{T}$ distribution is biased towards low values. Jets with $R=0.2$ restore the hard process $p_{T}$ distribution reasonably well, though there is still some PU contribution. Hereafter, we will mostly consider jets with $R=0.2$, $p_{T}>60 \mathrm{GeV}$ and $|\eta|<2.5$ for our analyses, unless stated otherwise.

\section{Dedicated LLP triggers based on L1 tracking}

In the previous section, we have discussed how the standard L1 triggers are not very efficient in selecting events from LLP scenario (A), where a pair of LLPs is directly produced and they decay into jets, especially when the LLP is light. In this section, we discuss some dedicated triggers based on information available at L1 for long-lived particles that can improve their signal efficiencies.

For long-lived particles, the trackless jet trigger was used in [48] as a High Level Trigger. Since jets coming from LLP decays will be displaced, these jets will have very few reconstructed tracks and will be mostly trackless. Their trigger design was to select events with jets having $E_{T}>25 \mathrm{GeV}$, which have no reconstructed tracks with $p_{T}>0.8 \mathrm{GeV}$ 

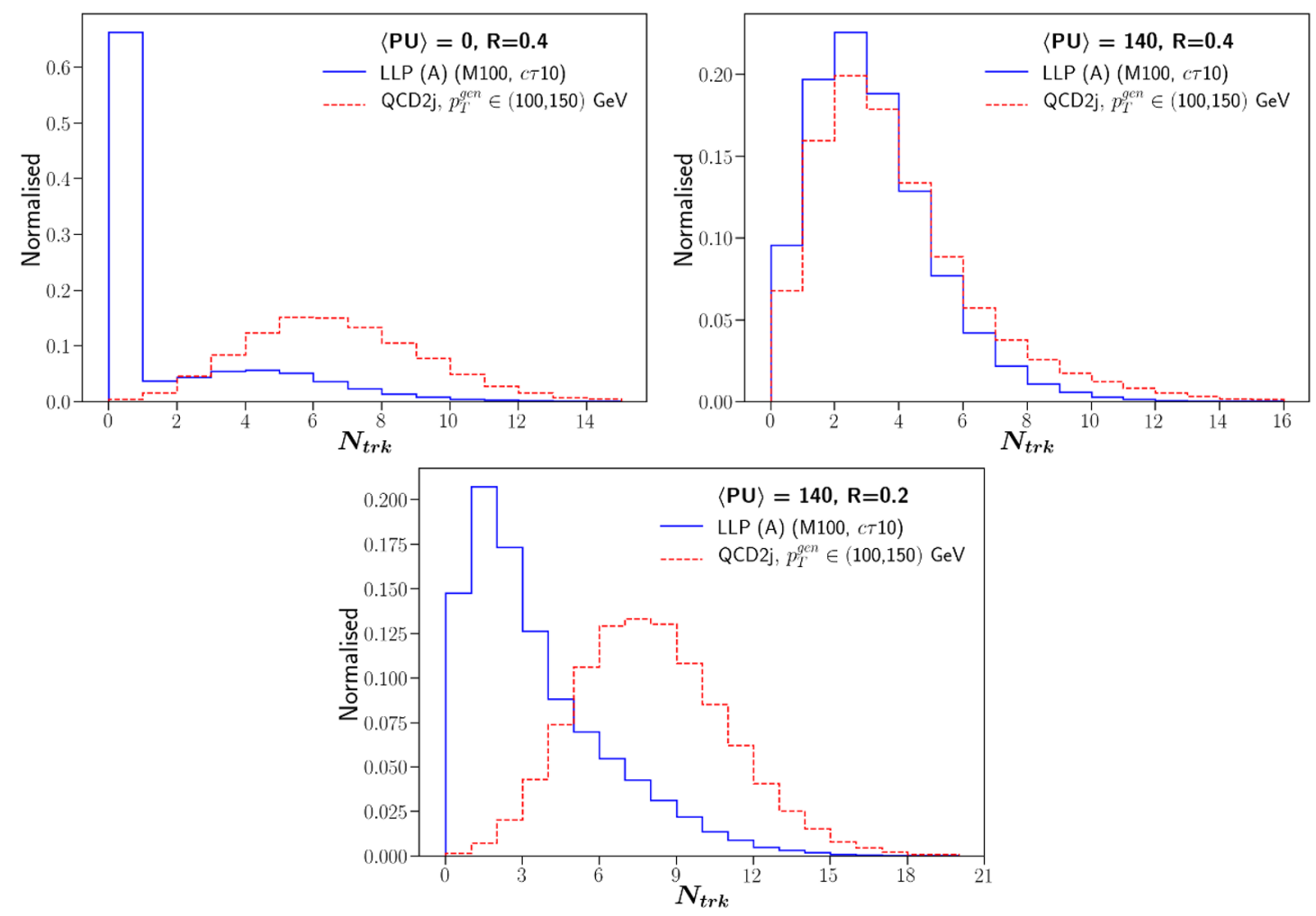

Figure 7. Distribution of number of tracks associated with jets clustered using anti- $k_{T}$ with $R=0.4$ coming from LLP benchmark $\left(M_{X}=100 \mathrm{GeV}\right.$ and $\left.c \tau=10 \mathrm{~cm}\right)$ from scenario (A) with zero PU (top left) and $140 \mathrm{PU}$ (top right) compared with the number of tracks associated with QCD jets. In the bottom plot, we have shown the distributions for jets with $R=0.2$ merged with 140 PU.

within $\Delta R=\sqrt{\Delta \eta^{2}+\Delta \phi^{2}}=0.2$ around the jet axis. ${ }^{11}$ They need an associated muon with $p_{T}>10 \mathrm{GeV}$ to pass the L1 trigger.

However, with the Phase-II upgrade, since tracking will be available at L1, we can think of designing some triggers for LLPs based on the tracking information at L1. However, we would only be able to have tracks for particles with $p_{T}>2 \mathrm{GeV}$ that are produced within a radial distance of $1 \mathrm{~cm}$ and half-length of $30 \mathrm{~cm}$. Long-lived particles, decaying after a radial distance of $1 \mathrm{~cm}$ or half-length of $30 \mathrm{~cm}$ won't have jets with tracks reconstructed at L1 unlike the QCD jets. Figure 7 (top left) shows the number of L1 tracks associated with a $R=0.4$ jet with $p_{T}>60 \mathrm{GeV}$, i.e., tracks within $\Delta R=0.4$ of the jet axis $\left(N_{\text {trk }}\right)$, for jets from LLP benchmark $\left(M_{X}=100 \mathrm{GeV}\right.$ and $\left.c \tau=10 \mathrm{~cm}\right)$ from scenario (A) and QCD jets, when there is no PU. We notice that they have significant difference.

However, with increasing the amount of PU to 140, the PU contribution dominates in both samples. As we can see from figure 3, the jet multiplicity in an event increases from around 3-4 to 10 on adding PU. Therefore, most of the jets in an event are PU jets and they control all the distributions in both signal and background. As a result, there is no difference between the $N_{\text {trk }}$ distribution for jets from LLP events and QCD ones. But once

\footnotetext{
${ }^{11}$ In this work, we denote the cone-size of an anti- $k_{T}$ clustered jet as $R$, and we use $\Delta R$ to denote some $\Delta \eta \times \Delta \phi$ region around the jet axis.
} 
we consider narrow jets of $\mathrm{R}=0.2$, as we have already discussed and demonstrated earlier, the number of jets coming only from PU decreases (compare figures 3 and 6 ). Therefore, $N_{\text {trk }}$ of a narrow $(R=0.2)$ jet again has some difference between LLP and QCD events, with the former shifted towards lesser number of tracks than the latter. The top right and bottom plots of figure 7 show the $N_{\text {trk }}$ distributions in the $140 \mathrm{PU}$ scenario with $R=0.4$ and $R=0.2$ jets respectively.

There is still some amount of PU contamination in the $N_{\text {trk }}$ distribution of $R=0.2$ jets which is evident from the comparison of the top left and bottom panels of figure 7 . Therefore, just using the $N_{\text {trk }}$ variable to discriminate between displaced and prompt jets won't be very efficient. We study many possible variables that can be constructed out of the tracking information at L1, and discuss how these variables and their correlations can help identifying displaced jets from prompt ones.

\subsection{Training a BDT to classify displaced and prompt jets based on tracking information}

We consider the following variables in addition to $N_{\text {trk }}$, which can help distinguish between jets coming from QCD dijet and LLP events:

- $\sum \mathbf{p}_{\mathrm{T}}$ of all tracks associated with a jet: for jets coming from LLP decays, the tracks associated with the jet will mostly be coming from PU. Therefore, we expect weaker correlation between the jet $p_{T}$ and the sum of $p_{T}$ of all the tracks associated with the jet $\left(\sum p_{T}=\sum_{i=1}^{N_{\text {trk }}} p_{T, i}\right)$ for an LLP jet than in a QCD prompt jet, where most of the tracks will actually be the constituents of the jet (see the correlation matrix of some of the track variables for signal and background in appendix A.2).

- Jet $\mathbf{z}$ vertex $\mathbf{z}_{\mathbf{j}_{-} \mathbf{v t x}}$ : the $z$-position of the jet vertex is defined as the $p_{T}$ weighted average of $z$-values of all the tracks associated with a jet $\left(z_{\mathrm{j}-\mathrm{vtx}}=\sum_{i=1}^{N_{\text {trk }}} p_{T, i} \times\right.$ $\left.z_{i} / \sum_{i=1}^{N_{\text {trk }}} p_{T, i}\right)[51]$.

- $\Delta \mathbf{z}_{\mathrm{j}_{-} \mathrm{vtx}}$ : we also calculate the $p_{T}$ weighted average of the difference between the $z$ values of tracks and the $z$-position of the jet vertex $\left(\Delta z_{\mathrm{j}_{\text {_vtx }}}=\sum_{i=1}^{N_{\text {trk }}} p_{T, i} \times \mid z_{i}-\right.$ $\left.z_{\mathrm{j}_{\text {vtx }}} \mid / \sum_{i=1}^{N_{\text {trk }}} p_{T, i}\right)$. This might give us an idea of how much the jet vertex is shifted along the $z$-direction due to stray PU tracks associated with an LLP or QCD jet, and we expect this to be smaller for the latter than the former.

- $\mathbf{p}_{\mathbf{T}(\mathrm{vtx})}^{\text {miss }}$ : we calculate a variable called $p_{T(\mathrm{vtx})}^{\text {miss }}$, which is the missing transverse momentum calculated for the vertex from which a jet is coming from. Say, a jet has the $z$ vertex position at $z_{\mathrm{j}_{-} \text {vtx }}$, and there are $n$ jets coming from the same $z$ vertex, within $1 \mathrm{~cm}$ (including the starting jet), then $p_{T(\mathrm{vtx})}^{\text {miss }}=\sqrt{\left(\sum_{n} p_{x}^{i}\right)^{2}+\left(\sum_{n} p_{y}^{i}\right)^{2}}$ where $p_{x}^{i}$ and $p_{y}^{i}$ are the $x$ and $y$ components of the momentum of the $i^{\text {th }}$ jet coming from the same vertex. The motivation for using such a variable is to capture the fact that QCD jets or jets from PU mostly satisfy the same $z$-vertex condition and therefore have very little values of $p_{T(\mathrm{vtx})}^{\mathrm{miss}}$, however, LLP jets hardly get associated to the same vertex, and are likely to have higher $p_{T(\mathrm{vtx})}^{\text {miss }}$ values. 
- $\mathbf{n}_{\mathbf{z}_{\text {trk_max }}}$ : next, we group all tracks associated with a jet having same $z$-positions (within $1 \mathrm{~mm}$ ) and label these different $z$-values as $z_{a}(a=1,2, \ldots)$, each having $n_{z_{a}}$ number of tracks, and $\sum_{z_{a}} n_{z_{a}}=N_{\mathrm{trk}}$. For each jet, the maximum number of tracks coming from the same $z$-position $\left(n_{z_{\text {trk_max }}}\right)$, is stored.

- $\Delta \mathbf{z}_{\text {trk_max }}$ : we calculate the average of the difference between the $z$-position with maximum number of tracks and all the other $z$-values weighted by the number of tracks coming from that particular $z$-value $\left(\Delta z_{\text {trk_max }}=\sum_{z_{a}} n_{z_{a}} \times\left|z_{a}-z_{\text {trk_max }}\right| / N_{\text {trk }}\right)$.

- $\sum \mathbf{p}_{\mathbf{T}}^{\mathbf{z}_{\text {trk max }}}, \sum \mathbf{p}_{\mathbf{T}}^{\mathbf{z}_{\mathbf{a}} \neq \mathbf{z}_{\text {trk_max }}}, \sum \mathbf{p}_{\mathbf{T}}^{\mathbf{z}_{\text {trk_max }}} / \sum \mathbf{p}_{\mathbf{T}}$ : also, the sum of $p_{T}$ of tracks coming from the $z$-position with maximum tracks and that of tracks coming from all other $z$-vertices are calculated separately as $\left(\sum p_{T}^{z_{\text {trk_max }}}=\sum_{i=1}^{n_{z_{\text {trk_max }}}} p_{T, i}\right)$ and $\left(\sum p_{T}^{z_{a} \neq z_{\text {trk_max }}}=\sum p_{T}-\sum p_{T}^{z_{\text {trk_max }}}\right)$ respectively. The fraction of $p_{T}$ coming from the $z$-vertex with maximum tracks in the total $p_{T}$ sum of all tracks associated with a jet $\left(\sum p_{T}^{z_{\text {trk_max }}} / \sum p_{T}\right)$ is also a variable of interest, which is expected to be closer to 1 for QCD prompt jets than LLP jets, because most of the tracks associated with a prompt QCD jet will come from the same $z$-position (within $1 \mathrm{~mm}$ ).

- Entropy variables: to quantify the spread of $z$-values of tracks associated with a jet, we also consider the Shannon entropy [67] of the $z$-position $\left(S\left(z_{i}\right)=-\sum_{i=1}^{N_{\text {trk }}} P\left(z_{i}\right) \times\right.$ $\left.\log _{N_{\text {trk }}} P\left(z_{i}\right)\right)$ as well as the $p_{T}\left(S\left(p_{T, i}\right)=-\sum_{i=1}^{N_{\text {trk }}} P\left(p_{T, i}\right) \log _{N_{\text {trk }}} P\left(p_{T, i}\right)\right)$ of the tracks associated with a jet. We consider different variants of these entropy variables - $S\left(\left|z_{i}\right| / \sum\left|z_{i}\right|\right), S\left(z_{i}+301\right)$ and $S\left(z_{i}+301 / \sum\left(z_{i}+301\right)\right)$ for the starting $z$ values of the tracks; and $S\left(p_{T, i}\right)$ and $S\left(p_{T, i} / \sum p_{T, i}\right)$ with the track $p_{T}$ values.

If the input of the Shannon entropy is a set of numbers with very less variation, the entropy value is close to 0 , and if the variation is maximum, the entropy value is closer to 1 . We expect for QCD prompt jets, most of the $\mathrm{L} 1$ tracks to come from the same $z$ positions unlike for displaced jets, which will mostly have tracks from PU associated with them, which will have different $z$ positions. The same might be true for $p_{T}$ of the tracks which will be more correlated for prompt jets.

- Variables with tracks within the narrow jet: we also calculate all the variables discussed above with tracks within $\Delta R=0.2$ of the jet axis (variables with a superscript saying 0.2).

- $\mathbf{N}_{\text {trk }} / \mathbf{N}_{\text {trk }}^{(\mathbf{0 . 2})}, \sum \mathbf{p}_{\mathbf{T}} / \sum \mathbf{p}_{\mathbf{T}}^{(\mathbf{0 . 2})}$ : finally, we also calculate the ratios $\frac{N_{\text {trk }}}{N_{\text {trk }}^{(0.2)}}$ and $\frac{\sum p_{T}}{\sum p_{T}^{(0.2)}}$.

Figure 8 shows the distributions of some of the track variables for two signal benchmark points from scenario (A) and for the background QCD dijet processes for three different $p_{T}$ regions at the generation level. We have shown distributions of all other variables in figures 18 and 19 in appendix A.1. The $N_{\mathrm{trk}}^{(0.2)}$ variable is similar to the $N_{\mathrm{trk}}$ variable, only with lesser number of tracks. The $p_{T(\mathrm{vtx})}^{\text {miss }}$ variable has a longer tail for the LLP jets. This is due to the fact that the LLP jets won't have much associated L1 tracks and they are not identified to come from the same $z$-vertex. Therefore, a vertex will mostly have a single jet associated to it, and there will be missing transverse energy at the vertex. The $p_{T}$ threshold 

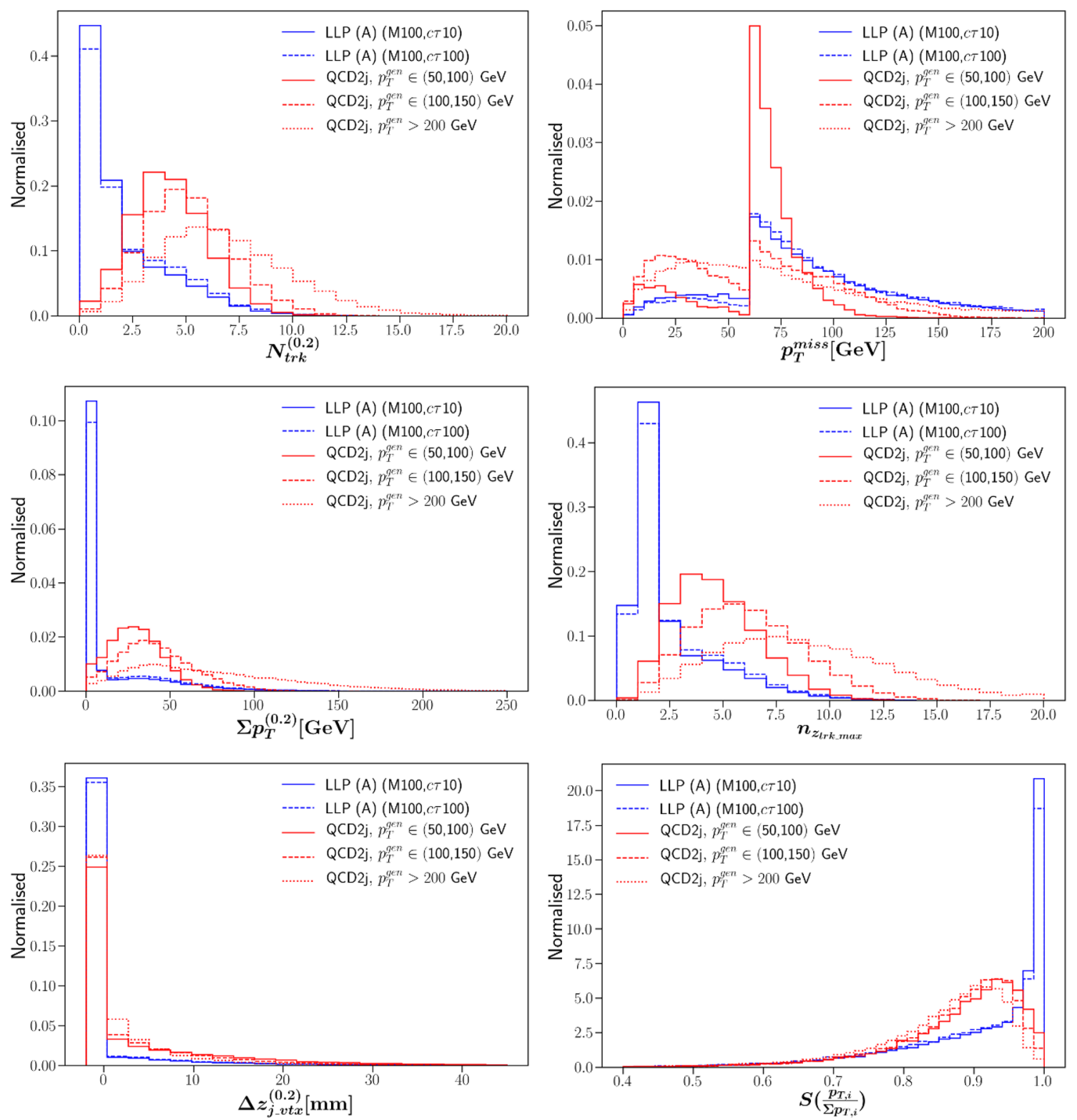

Figure 8. Distributions for some of the track variables for two signal points from LLP scenario (A) and QCD dijet processes with different $p_{T}$ cuts at the parton level.

for jets is $60 \mathrm{GeV}$ in our case and therefore, there is a peak around that value, since if a single jet is identified with a vertex and its $p_{T}$ is $60 \mathrm{GeV}$, the missing transverse momentum associated with that vertex will be $60 \mathrm{GeV}$ only. The entropy variable $S\left(p_{T, i} / \sum p_{T, i}\right)$ peaks around 1 for the signal since the LLP jets have mostly PU tracks which have a wide distribution of $p_{T}$ values.

As we can see from figure 8 , no single variable is very powerful in discriminating between the signal and the background, however, their correlations do have some differences, as we can see in figures 20 and 21 in appendix A.2. We, therefore, train a BDT classifier using the TMVA[68] framework of ROOT[69] with all these variables along with the $p_{T}$ and $\eta$ 

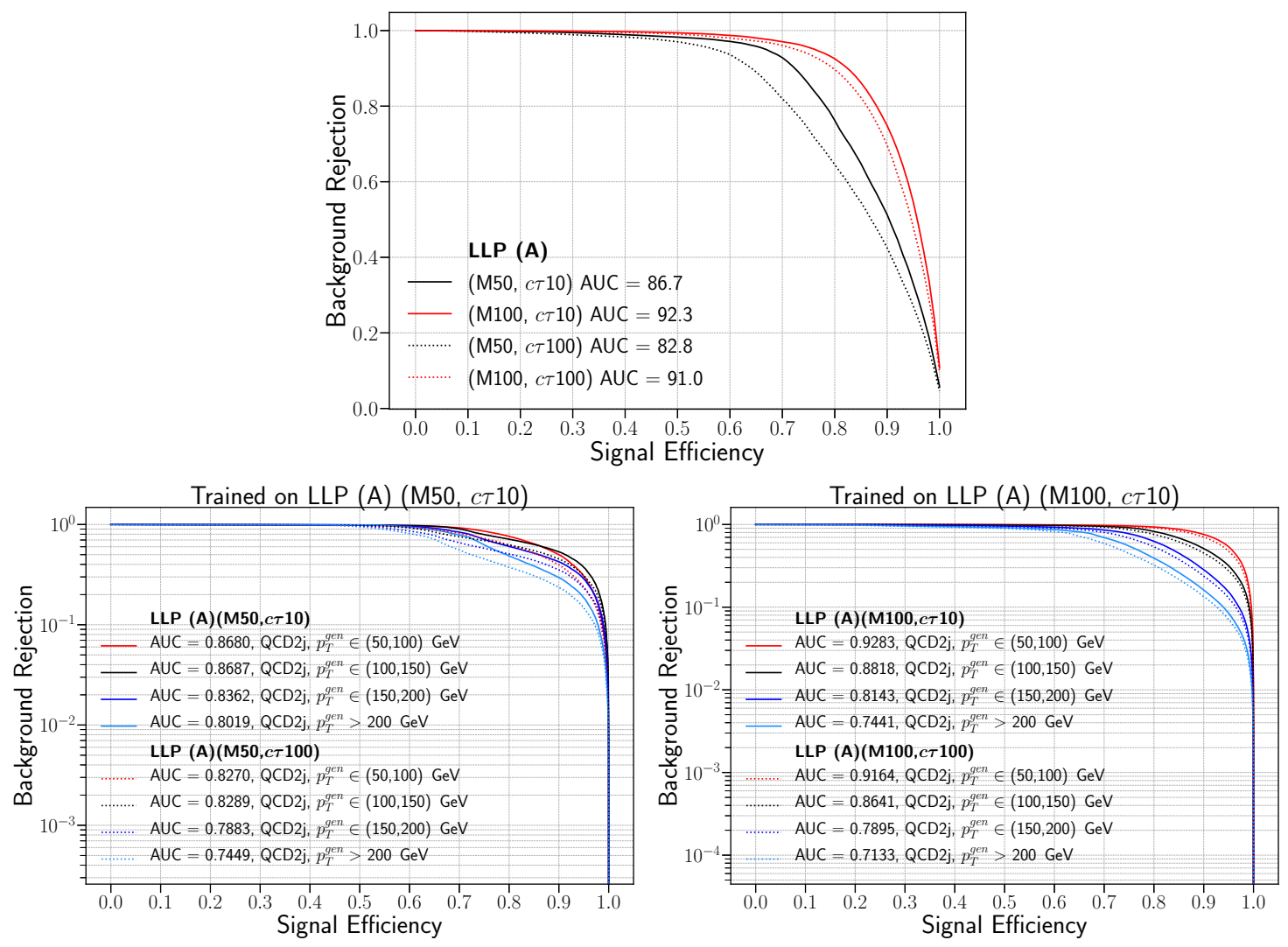

Figure 9. ROC curves for selecting LLP jets for two points from BP1 $-M_{X}=100 \mathrm{GeV}, c \tau=$ $10 \mathrm{~cm}(\mathrm{M} 100, \mathrm{DL} 10)$ and $M_{X}=50 \mathrm{GeV}, c \tau=10 \mathrm{~cm}$ (M50,DL10) from QCD dijet samples with different $p_{T}$ cuts at the parton level.

of the jets for the LLP benchmark points from scenario (A) as signals and the background being a merged sample of QCD dijet processes with $p_{T}$ between $50-100 \mathrm{GeV}, 100-150 \mathrm{GeV}$, $150-200 \mathrm{GeV}$ and greater than $200 \mathrm{GeV}$ at the generation level, with appropriate weights according to their cross-sections respectively. Out of all the variables that we use for training, following is the list of the top five variables, which are most efficient in discriminating between the signal and the background:

$$
p_{T(\mathrm{vtx})}^{\mathrm{miss}}, \quad n_{z_{\text {trk } \max }}, \quad N_{\text {trk }}, \quad \sum p_{T}^{(0.2)}, \quad \Delta z_{\text {j_vtx }}^{(0.2)}
$$

The top panel of figure 9 shows the ROC curves for classifying the signal and background using tracking variables. We now train the BDT on a particular mass value and decay length and apply the training on that benchmark point as well as the benchmark points with the same mass and a different decay length. The bottom plots of figure 9 show the application ROC curves for the benchmark points from scenario (A) when trained on

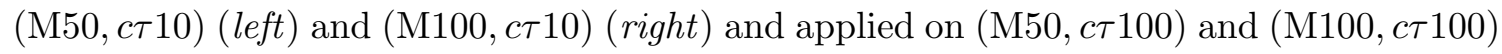
respectively. The application ROCs are shown for jets from each of the $p_{T}^{\text {gen }}$ bins of the background QCD dijet events. 


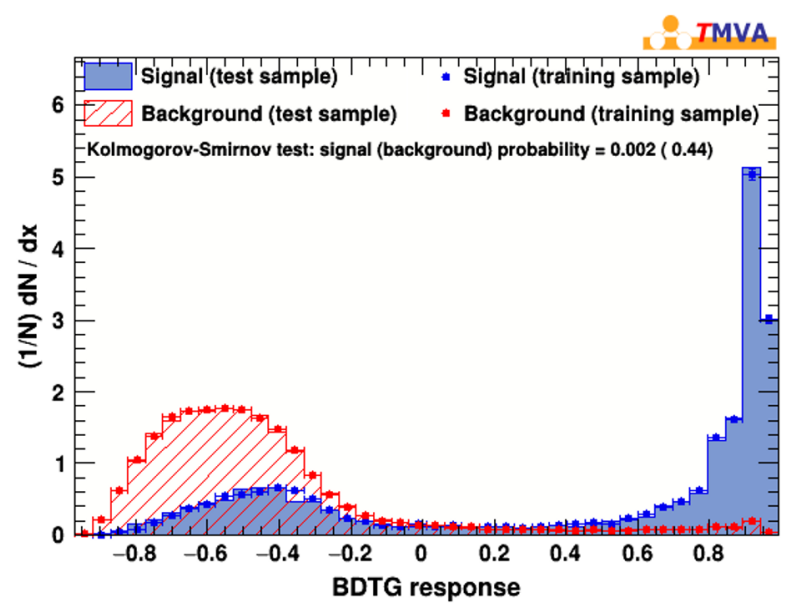

Figure 10. Distributions of the BDT scores of signal jets coming from LLP benchmark point with mass $50 \mathrm{GeV}$ and decay length $10 \mathrm{~cm}$ from scenario (A) and background jets from merged dijet process with different $p_{T}^{\text {gen }}$ bins.

Even after considering $R=0.2$ jets, we have not been able to eliminate the PU contribution completely. There might be jets with $p_{T}>60 \mathrm{GeV}$ which come from the PU in the LLP events. These jets are similar to prompt QCD jets, and we therefore expect that the BDT classifier won't be able to discriminate between these jets. In figure 10, we show the BDT score distributions for LLP benchmark point with mass $50 \mathrm{GeV}$ and decay length $10 \mathrm{~cm}$ from scenario (A) as the signal and the merged sample of dijet processes with different $p_{T}^{\text {gen }}$ cuts as the background. We find that a small part of the signal BDT score distribution is similar to that of the background which are the remnant PU jets in the signal sample, and therefore, have similar BDT scores as the QCD jets background.

\subsection{Triggers based on the BDT training using variables from tracking}

We now discuss some triggers constructed using the above BDT classification and quote the signal efficiency and background rates for each of them. In the Phase-II upgrade, the L1 trigger hardware will have FPGAs which will be able to handle small scale machine learning (ML) applications, like BDT classification [70, 71]. We choose three points from the ROC curves, corresponding to $98 \%, 90 \%$ and $70 \%$ background rejections. Following are the different trigger cuts we apply for selecting the signal LLP events from the background QCD prompt jets:

- $\mathbf{T}_{\mathbf{1}}$ : at least one $R=0.2$ jet with $p_{T}>60 \mathrm{GeV}$;

- $\mathbf{T}_{2}^{\mathbf{a}}: T_{1}+$ that jet passes the BDT threshold corresponding to a background rejection of $98 \%(a=0), 90 \%(a=1)$ and $70 \%(a=2)$;

- $\mathbf{T}_{3}^{\mathbf{a}}: T_{1}+$ no other jet from the same $z$-vertex (i.e., $\Delta z$ with all other jets is greater than $1 \mathrm{~cm})+T_{2}^{a}$

- $\mathbf{T}_{\mathbf{4} \mathbf{b}}^{\mathbf{a}}$ : at least one jet with $p_{T} \in(60,100)(b=1) / p_{T} \in(60,120)(b=2)+$ no other jet from the same $z$-vertex (i.e., $\Delta z$ with all other jets is greater than $1 \mathrm{~cm}$ ) $+T_{3}^{a}$; 
$T_{1}$ is the efficiency of selecting events containing at least one jet with $p_{T}$ greater than $60 \mathrm{GeV}$. Since the LLP jets are narrower than QCD jets (due to late decays), their $p_{T}$ distribution is not affected much when we consider $R=0.2$ jets. However, low $p_{T}$ QCD jets will be much more affected as we have discussed earlier. We then choose a point from the ROC curve corresponding to a particular background rejection, and use the BDT threshold corresponding to that point as a cut on the jet's BDT score. If an event has at least one jet satisfying the $p_{T}$ condition and having a BDT score above the threshold, we select the event as a signal and this is our $T_{2}^{a}$ trigger. For $T_{3}^{a}$ trigger, we have an added condition that our trigger jet (passing $T_{1}$ and $T_{2}^{a}$ ) shall not have any other jet coming from a $z$-vertex which is within $1 \mathrm{~cm}$ of the trigger jet's $z$-vertex position. We expect this condition to affect the QCD jets more since they usually have same $z$-vertex unlike the LLP jets which mostly won't have many reconstructed L1 tracks, and hence mostly, they will not come from the same $z$-vertex (as we already saw in the standard same vertex multijet trigger efficiencies quoted for zero PU in table 2). Finally, we define another trigger $T_{4 b}^{a}$ which demands the transverse momentum of jets to be within particular $p_{T}$ ranges. As seen from figure 9, the ROC performance degrades when training is applied on QCD jets with higher $p_{T}^{\text {gen }}$ bins, and therefore, putting an upper limit on the jet $p_{T}$ can reduce background from higher $p_{T}^{\text {gen }}$ bins.

An efficient LLP trigger design must be such that it has high signal efficiency and the rates are comparable to that of other triggers, ensuring that the total rate does not exceed the allowed trigger bandwidth. We now discuss the calculation of trigger rates, which will be dominated by background processes due to their huge cross section.

The trigger rate (in $\mathrm{Hz}$ ) from background is calculated as follows:

$$
\mathcal{R}_{B}=\sigma(\mathrm{nb}) \times \mathcal{L}\left(\mathrm{nb}^{-1} \mathrm{~Hz}\right) \times \epsilon_{B}
$$

where $\sigma$ is the background cross section (in nb), $\mathcal{L}$ is the peak instantaneous luminosity (in $\mathrm{nb}^{-1} \mathrm{~Hz}$ ) and $\epsilon_{B}$ is the efficiency of the triggers to select a background event. For the HL-LHC, $\mathcal{L}=5.6 \times 10^{34} \mathrm{~cm}^{-2} \mathrm{~s}^{-1}=56 \mathrm{nb}^{-1} \mathrm{~Hz}$ for the $140 \mathrm{PU}$ scenario. For example, the $\mathrm{QCD}$ dijet rate for $p_{T}^{\text {gen }} \in(50,100) \mathrm{GeV}$ is:

$$
\mathcal{R}_{B}=1.868 \times 10^{4} \mathrm{nb} \times 56 \mathrm{nb}^{-1} \mathrm{~Hz} \sim 1046 \mathrm{kHz} .
$$

We use the leading order PYTHIA cross sections for calculating the rates. The higher order corrections can contribute to another factor of $\sim 2 .{ }^{12}$ We have to multiply this huge rate by $\epsilon_{B}$, which is the trigger efficiencies for background events, and which will quantify how much of this rate can we reject using our triggers.

Tables 3 and 4 show the trigger efficiencies for different LLP signal benchmark points from scenario (A) and the background rates for different $p_{T}^{\text {gen }}$ bins for a background rejection of $98 \%$ and $70 \%$ (in each of the $p_{T}^{\text {gen }}$ bins) respectively. A similar table, table 8 , for a background rejection of $90 \%$ has been given in appendix A.3. We find that the trigger rates for $p_{T}^{\text {gen }} \in(50,100) \mathrm{GeV}$ can be reduced to about $5.4-6.4 \mathrm{kHz}\left(T_{41}^{0}\right.$ column of table 3$)$ from

\footnotetext{
${ }^{12}$ The other single jet backgrounds can be $Z+$ jets or $t \bar{t}$, where the cross sections are few thousand pb, which is very small compared to the dijet cross sections for low $p_{T}^{\text {gen }}$ bins.
} 


\begin{tabular}{|c|c|c|c|c|c|c|}
\hline LLP & $\begin{array}{c}\text { QCD } 2 \mathrm{j} \\
p_{T}^{\text {gen }}[\mathrm{GeV}] \\
\left(\mathcal{R}_{B}[\mathrm{kHz}]\right)\end{array}$ & $\begin{array}{c}T_{1} \\
\mathcal{R}_{B}[\mathrm{kHz}] \\
\left(\epsilon_{S}[\%]\right)\end{array}$ & $\begin{array}{c}T_{2}^{0} \\
\mathcal{R}_{B}[\mathrm{kHz}] \\
\left(\epsilon_{S}[\%]\right)\end{array}$ & $\begin{array}{c}T_{3}^{0} \\
\mathcal{R}_{B}[\mathrm{kHz}] \\
\left(\epsilon_{S}[\%]\right)\end{array}$ & $\begin{array}{c}T_{41}^{0} \\
\mathcal{R}_{B}[\mathrm{kHz}] \\
\left(\epsilon_{S}[\%]\right)\end{array}$ & $\begin{array}{c}T_{42}^{0} \\
\mathcal{R}_{B}[\mathrm{kHz}] \\
\left(\epsilon_{S}[\%]\right)\end{array}$ \\
\hline \multirow{4}{*}{$\begin{array}{c}M=50 \mathrm{GeV} \\
c \tau=10 \mathrm{~cm}\end{array}$} & $50,100(1046)$ & $301.5(23.43)$ & $7.2(13.29)$ & 7 (13.18) & $6.4(10.68)$ & $6.7(11.91)$ \\
\hline & $100,150(53.4)$ & $46.4(23.43)$ & $1.5(14.84)$ & $1.3(14.64)$ & $0.7(12.21)$ & $0.9(13.39)$ \\
\hline & $150,200(7.5)$ & $7.3(23.43)$ & $0.3(14.15)$ & $0.2(14.01)$ & $0.06(11.54)$ & $0.08(12.75)$ \\
\hline & $>200(2.7)$ & $2.7(23.43)$ & $0.1(13.97)$ & $0.08(13.84)$ & $0.02(11.37)$ & $0.02(12.58)$ \\
\hline \multirow{4}{*}{$\begin{aligned} M & =50 \mathrm{GeV} \\
c \tau & =100 \mathrm{~cm}\end{aligned}$} & $50,100(1046)$ & $301.5(18.34)$ & $7.2(8.55)$ & $7(8.48)$ & $6.4(6.69)$ & $6.7(7.47)$ \\
\hline & $100,150(53.4)$ & $46.4(18.34)$ & $1.5(9.92)$ & $1.3(9.79)$ & $0.7(7.90)$ & $0.9(8.71)$ \\
\hline & $150,200(7.5)$ & $7.3(18.34)$ & $0.3(9.33)$ & $0.2(9.23)$ & $0.06(7.39)$ & $0.08(8.19)$ \\
\hline & $>200(2.7)$ & $2.7(18.34)$ & $0.1(9.15)$ & $0.08(9.06)$ & $0.02(7.23)$ & $0.02(8.03)$ \\
\hline \multirow{4}{*}{$\begin{array}{c}M=100 \mathrm{GeV} \\
c \tau=10 \mathrm{~cm}\end{array}$} & $50,100(1046)$ & $301.5(82.38)$ & $7(61.80)$ & $6.7(60.20)$ & $5.4(37.15)$ & $5.9(46.59)$ \\
\hline & $100,150(53.4)$ & $46.4(82.38)$ & $1.4(53.89)$ & $1.2(52.64)$ & $0.3(28.14)$ & $0.5(38.12)$ \\
\hline & $150,200(7.5)$ & $7.3(82.38)$ & $0.3(35.40)$ & $0.2(34.66)$ & $0.0(7.41)$ & $0.01(16.71)$ \\
\hline & $>200(2.7)$ & $2.7(82.38)$ & $0.1(25.46)$ & $0.08(25.05)$ & $0.0(1.95)$ & $0.0(6.25)$ \\
\hline \multirow{4}{*}{$\begin{array}{c}M=100 \mathrm{GeV} \\
c \tau=100 \mathrm{~cm}\end{array}$} & $50,100(1046)$ & $301.5(68.84)$ & $7(48.32)$ & $6.7(46.40)$ & $5.4(26.31)$ & $5.9(33.14)$ \\
\hline & $100,150(53.4)$ & $46.4(68.84)$ & $1.4(41.10)$ & $1.2(39.54)$ & $0.3(19.11)$ & $0.5(26.15)$ \\
\hline & $150,200(7.5)$ & $7.3(68.84)$ & $0.3(25.54)$ & $0.2(24.67)$ & $0.0(4.66)$ & $0.01(10.71)$ \\
\hline & $>200(2.7)$ & $2.7(68.84)$ & $0.1(17.91)$ & $0.08(17.43)$ & $0.0(1.36)$ & $0.0(4.07)$ \\
\hline
\end{tabular}

Table 3. Efficiency of selecting QCD and LLP events for LLP scenario (A) benchmark points with the modified trackless trigger at L1 for Phase-II. BDT cut is applied considering $98 \%$ background rejection. Quantity in parenthesis represents corresponding signal efficiency.

$1046 \mathrm{kHz}$ for a signal efficiency of $\sim 11 \%$ for $(\mathrm{M} 50, c \tau 10)$ and $\sim 37 \%$ for $(\mathrm{M} 100, c \tau 10)$. The actual rates will be twice if we consider higher orders, and therefore, the final background rates reduce to about $10-12 \mathrm{kHz}$.

For $70 \%$ background rejection, the background rates are really high $(\sim 2 \times 86 \mathrm{kHz})$, and the signal efficiencies increase by few percentage. So if we are limited by rates, choosing a $98 \%$ or $90 \%$ background rejection point from the ROC will be more useful, because they have low background rates and there is little compromise on the signal efficiencies.

The BDT training, here, was done using a merged background sample of QCD dijet events with different $p_{T}^{\text {gen }}$ cuts weighted according to their cross-section. We could also train the BDT using a categorical training with multiclass approach with each $p_{T}$ bin serving as separate backgrounds. It will improve the performance of the application ROCs (bottom panel plots of figure 9) for the higher $p_{T}^{\text {gen }}$ bins, which are now poorer than the $p_{T}^{\text {gen }} \in(50,100) \mathrm{GeV}$ bin because it has the highest cross section and the highest weight in the training. We here present a conservative analysis, and the rates of the higher $p_{T}$ bins are very low to start with. Also, using the upper cut on $p_{T}$ values of jets suppresses the dijet background from higher $p_{T}^{\text {gen }}$ bins.

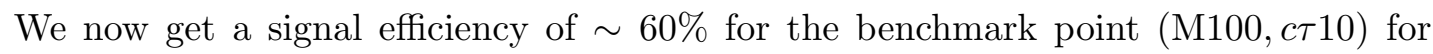
a background rejection of $98 \%$, which is quite an improvement compared to the signal efficiency of this benchmark point when standard triggers are used $(\sim 27 \%, \sim 5 \%$ and 


\begin{tabular}{|c|c|c|c|c|c|}
\hline $\operatorname{LLP}(\mathrm{A})$ & $\begin{array}{c}\text { QCD } 2 \mathrm{j} \\
p_{T}^{\mathrm{gen}}[\mathrm{GeV}] \\
\left(\mathcal{R}_{B}[\mathrm{kHz}]\right)\end{array}$ & $\begin{array}{c}T_{2}^{2} \\
\mathcal{R}_{B}[\mathrm{kHz}] \\
\left(\epsilon_{S}[\%]\right)\end{array}$ & $\begin{array}{c}T_{3}^{2} \\
\mathcal{R}_{B}[\mathrm{kHz}] \\
\left(\epsilon_{S}[\%]\right)\end{array}$ & $\begin{array}{c}T_{41}^{2} \\
\mathcal{R}_{B}[\mathrm{kHz}] \\
\left(\epsilon_{S}[\%]\right)\end{array}$ & $\begin{array}{c}T_{42}^{2} \\
\mathcal{R}_{B}[\mathrm{kHz}] \\
\left(\epsilon_{S}[\%]\right)\end{array}$ \\
\hline \multirow{4}{*}{$\begin{array}{c}M=50 \mathrm{GeV} \\
c \tau=10 \mathrm{~cm}\end{array}$} & $50,100(1046)$ & $103.2(19.79)$ & $95(19.28)$ & $86.1(16.77)$ & $93.1(18.11)$ \\
\hline & $100,150(53.4)$ & $19.2(19.36)$ & $13.4(18.87)$ & $5.7(16.34)$ & $10.6(17.69)$ \\
\hline & $150,200(7.5)$ & $3.3(18.06)$ & $1.6(17.67)$ & $0.2(15.08)$ & $0.6(16.46)$ \\
\hline & $>200(2.7)$ & $1.2(17.58)$ & $0.4(17.23)$ & $0.05(14.61)$ & $0.08(16.01)$ \\
\hline \multirow{4}{*}{$\begin{aligned} M & =50 \mathrm{GeV} \\
c \tau & =100 \mathrm{~cm}\end{aligned}$} & $50,100(1046)$ & $103.2(14.48)$ & $95(14.03)$ & $86.1(11.63)$ & $93.1(12.78)$ \\
\hline & $100,150(53.4)$ & $19.2(14.08)$ & $13.4(13.65)$ & $5.7(11.24)$ & $10.6(12.40)$ \\
\hline & $150,200(7.5)$ & $3.3(12.94)$ & $1.6(12.58)$ & $0.2(10.15)$ & $0.6(11.31)$ \\
\hline & $>200(2.7)$ & $1.2(12.50)$ & $0.4(12.17)$ & $0.05(9.76)$ & $0.08(10.89)$ \\
\hline \multirow{4}{*}{$\begin{array}{c}M=100 \mathrm{GeV} \\
c \tau=10 \mathrm{~cm}\end{array}$} & $50,100(1046)$ & $100.5(77.73)$ & $87(72.90)$ & $77.6(52.11)$ & $85.2(61.72)$ \\
\hline & $100,150(53.4)$ & $19.4(73.28)$ & $11.5(69.56)$ & $3.7(47.56)$ & $8.6(57.81)$ \\
\hline & $150,200(7.5)$ & $3.6(69.24)$ & $1.3(66.49)$ & $0.1(44.18)$ & $0.2(53.94)$ \\
\hline & $>200(2.7)$ & $1.4(64.27)$ & $0.3(62.41)$ & $0.02(39.85)$ & $0.03(49.09)$ \\
\hline \multirow{4}{*}{$\begin{array}{c}M=100 \mathrm{GeV} \\
c \tau=100 \mathrm{~cm}\end{array}$} & $50,100(1046)$ & $100.5(64.02)$ & $87(59.53)$ & $77.6(39.17)$ & $85.2(47.27)$ \\
\hline & $100,150(53.4)$ & $19.4(59.83)$ & $11.5(56.16)$ & $3.7(35.01)$ & $8.6(43.47)$ \\
\hline & $150,200(7.5)$ & $3.6(55.60)$ & $1.3(52.72)$ & $0.1(31.97)$ & $0.2(39.52)$ \\
\hline & $>200(2.7)$ & $1.4(50.09)$ & $0.3(48.53)$ & $0.02(28.49)$ & $0.03(35.28)$ \\
\hline
\end{tabular}

Table 4. Efficiency of selecting QCD and LLP events for LLP scenario (A) benchmark points with the modified trackless trigger at L1 for Phase-II. BDT cut is applied considering $70 \%$ background rejection. Quantity in parenthesis represents corresponding signal efficiency.

$\sim 14 \%$ with single jet, dijet and quad jet triggers respectively). The total signal efficiency for the benchmark point (M50, $c \tau 10$ ) is $\sim 13 \%$ from the standard single jet, dijet and quad jet triggers (from table 2). From the track-based triggers that we have defined in this section, we get at best a signal efficiency of $19.79 \%$ for $70 \%$ background rejection for the same benchmark point. This might not seem much of an improvement unless we are selecting different events from the standard triggers and the one defined by us.

It will be, therefore, interesting to check the exclusiveness of the events selected by these dedicated LLP triggers, that we define here, and the ones selected by standard L1 single jet, dijet and quad jet triggers. The overlap between the events selected with standard triggers and the dedicated triggers defined in this work is just $5 \%$ for the benchmark point (M50, c 110$)$ in scenario (A). Therefore, they are mostly selecting different events, and that is because, the standard triggers are mostly triggered by pile-up jets, and our defined triggers are mostly triggered by jets from LLPs. Therefore, we can select an additional $\sim 14 \%$ of signal events even for the LLP of mass $50 \mathrm{GeV}$ and decay length $10 \mathrm{~cm}$ with the dedicated LLP triggers, making the total signal efficiency close to $\sim 27 \%$.

Also, for lower mass LLPs, the signal efficiency is mostly affected due to the first cut only, $T_{1}$. It would be important to lower the $p_{T}$ threshold to increase sensitivity for such 
lighter LLPs. However, decreasing the $p_{T}$ threshold will increase the PU contamination drastically, even when we consider narrow jets of $R=0.2$. In that case, we will need much more sophisticated techniques to deal with PU at lower $p_{T}$ thresholds, which is outside the scope of the present work.

We now discuss some other LLP scenarios and check the performance of the above discussed tracking variables in the context of these different LLP scenarios.

\section{Performance of the classifiers based on tracking for some other LLP scenarios}

We have discussed the prospects of using various tracking variables to design some dedicated triggers at L1 for the LLP scenario where a pair of LLPs is directly produced and then decay to jets. In this section, we extend our analysis to some other LLP scenarios and briefly discuss the findings. We have chosen each of these scenarios with some motivation to discuss slightly varying aspects of LLPs.

\subsection{Scenario with direct pair-production of LLPs and their decay to jets and invisible particle}

We started with the direct production of LLPs in quark-initiated processes and their further decay into jets. Within the same production mechanism of LLPs, we now consider a different decay mode. The LLP can decay into jets and invisible particles. In cases of prompt decay, such events will be characterised by high missing transverse energies $\left(\mathbb{E}_{T}\right)$ due to the presence of the invisible particles in the final state. However, if such decays are displaced, the $\mathbb{E}_{T}$ distribution might be different. Therefore, we are interested to study such a benchmark to have an idea of the efficiency of the standard $\mathbb{E}_{T}$ trigger at L1 for LLPs decaying into one or more invisible particles. Following are the simulation details for this scenario, which will hereafter be referred to as LLP scenario (B).

$$
\text { (B) } \mathbf{p p} \rightarrow \mathbf{X X}, \quad \mathbf{X} \rightarrow \mathbf{Y j j} \text {. }
$$

We generate this using PYTHIA6 similar to scenario (A) where a pair of LLPs are directly produced and they decay to an invisible particle and two quarks, which hadronise to give jets. We study two different mass points of the invisible particle $-300 \mathrm{GeV}$, and $400 \mathrm{GeV}$, with the LLP mass fixed to $500 \mathrm{GeV}$, and having two different proper decay lengths of $10 \mathrm{~cm}$ and $100 \mathrm{~cm}$ each. The LLP is assumed to decay to the aforementioned final state with $100 \%$ branching.

Let us first start by looking at the $\mathbb{E}_{T}$ distribution of these benchmarks to see how it gets affected for displaced cases. We calculate $\mathbb{E}_{T}$ using tracks $\left(\mathbb{E}_{T}(\right.$ trk $\left.)\right)$ reconstructed at L1 which start from the primary vertex. ${ }^{13}$ Figure 11 shows the distribution of $\mathbb{E}_{T}$ (trk) for the particle $X$ having a mass of $500 \mathrm{GeV}$, decaying into jets and a missing particle of mass $300 \mathrm{GeV}$ and $400 \mathrm{GeV}$.

\footnotetext{
${ }^{13}$ The primary vertex is identified at L1 as the vertex with the maximum value of $\sum p_{T}^{2} / n_{z_{a}}$, where $n_{z_{a}}$ is the total number of $\mathrm{L} 1$ tracks associated with the vertex.
} 

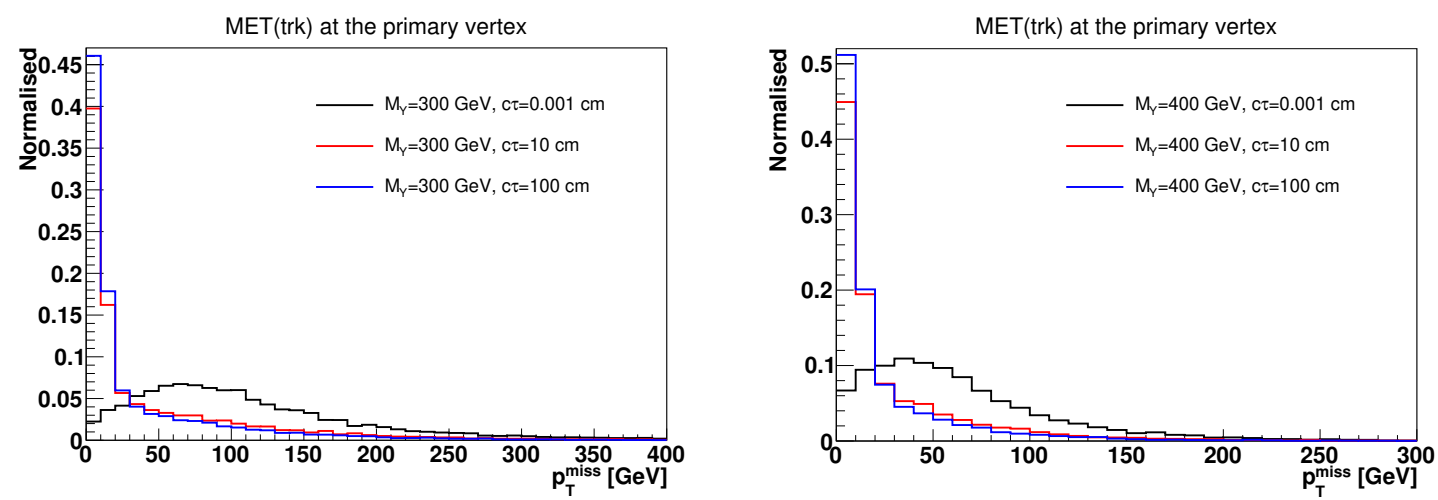

Figure 11. Normalised distribution of $\mathbb{E}_{T}($ trk $)$ calculated using L1 tracks from the identified primary vertex (PV) for prompt decay as well as for decay length values of $10 \mathrm{~cm}$ and $100 \mathrm{~cm}$ of particle $X$ of mass $500 \mathrm{GeV}$ with the mass of the invisible particle $\left(M_{Y}\right)$ being $300 \mathrm{GeV}$ (left) and $400 \mathrm{GeV}$ (right).

From figure 11, we find that the $\mathbb{E}_{T}$ (trk) distribution for prompt decay of $X$ does have a longer tail. However, when the LLP has a decay length of the order of few $\mathrm{cm}$, there are no L1 tracks and as we have already discussed, the primary vertex identification is not correct. Most of the time, PU vertices get identified as primary vertex instead of the hard process and therefore, most of the events have very low values of $\mathbb{E}_{T}$. Thus, standard $\mathbb{E}_{T}$ triggers won't be efficient in selecting LLP events even if they decay into final states involving invisible particles.

We now want to check how efficient L1 track variables are in selecting events from this scenario. Figure 12 shows the classification ROC curve for jets coming from LLPs from different benchmark points of scenario (B) as signal and QCD prompt jets (merged sample of all $p_{T}^{\text {gen }}$ bins) as background using L1 track variables. The benchmark point from scenario (B) where mass of the invisible particle is $400 \mathrm{GeV}$ (and $M_{X}=500 \mathrm{GeV}$ ) is similar to the scenario (A) benchmark point where the LLP mass is $100 \mathrm{GeV}$ - in both cases the jets will have similar boost distribution. However, in this case, the boost of the LLP will be smaller since it has higher mass, and therefore, it decays more often within the tracker than in scenario (A). The ROC performance is, therefore, slightly better.

\subsection{Scenario where LLPs are produced from the decay of Higgs boson and decay to jets}

LLPs can also be produced from the decay of an on-shell resonance, in which case the boost of the long-lived particles depend on the mass of the intermediate particle produced, in addition to its own mass. We now study this different type of production mode of LLPs, where they are produced from the decay of the SM Higgs boson, where the latter is produced in gluon-initiated process. Later we also discuss briefly the case where the LLPs come from the decay of a heavy resonance, which can be a heavy Higgs boson or any other BSM particle. We consider the decay of LLPs into quarks only.

$$
\text { (C) } \mathbf{p p} \rightarrow \mathbf{h} \rightarrow \mathbf{X X}, \quad \mathbf{X} \rightarrow \mathbf{j j} \text {. }
$$




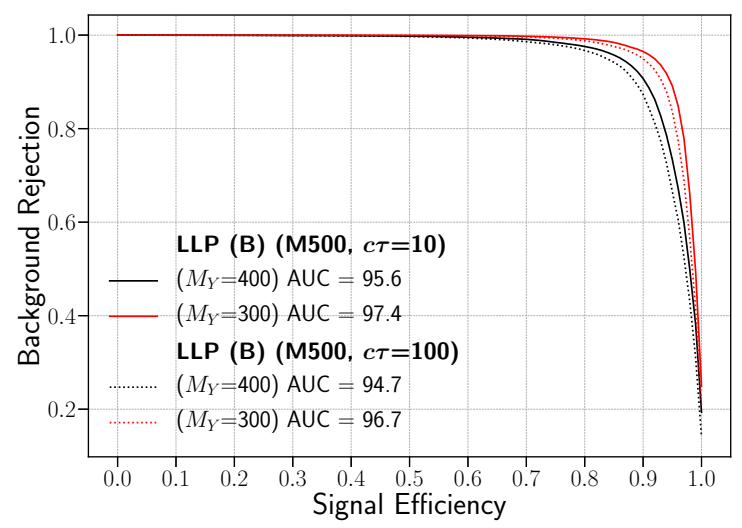

Figure 12. ROC curves for selecting displaced jets coming from LLP decay from prompt QCD ones for four benchmark points from scenario (B) with mass of the LLP $M_{X}=500 \mathrm{GeV}$ having decay lengths $10 \mathrm{~cm}$ and $100 \mathrm{~cm}$ using tracking variables. Two different masses of the invisible particle $300 \mathrm{GeV}$ and $400 \mathrm{GeV}$ are considered.

\begin{tabular}{|c|c|c|c|c|c|c|c|}
\hline $\begin{array}{c}\text { Mass }[\mathrm{GeV}], \\
\text { Decay Length }[\mathrm{cm}]\end{array}$ & $\begin{array}{c}\text { Reco as } \\
\text { L1 tracks }\end{array}$ & $\begin{array}{c}\text { Before } \\
\text { MTD }\end{array}$ & $\begin{array}{c}\text { Before } \\
\text { ECAL }\end{array}$ & $\begin{array}{c}\text { Before } \\
\text { HCAL }\end{array}$ & $\begin{array}{c}\text { Before } \\
\text { MS }\end{array}$ & $\begin{array}{c}\text { Inside } \\
\text { MS }\end{array}$ & $\begin{array}{c}\text { Outside } \\
\text { detector }\end{array}$ \\
\hline 10,10 & 2.44 & 77.23 & 2.89 & 5.96 & 5.24 & 3.87 & 2.37 \\
10,100 & 0.25 & 18.28 & 1.90 & 6.14 & 16.06 & 18.40 & 38.98 \\
30,10 & 7.70 & 88.34 & 0.78 & 1.19 & 1.02 & 0.77 & 0.20 \\
30,100 & 0.85 & 44.58 & 3.56 & 9.71 & 16.28 & 12.32 & 12.71 \\
50,10 & 17.23 & 81.26 & 0.37 & 0.48 & 0.40 & 0.24 & 0.02 \\
50,100 & 1.97 & 64.92 & 3.53 & 7.27 & 8.20 & 7.70 & 6.41 \\
\hline
\end{tabular}

Table 5. Fraction of decays for scenario (C) in various detector parts.

We generate the Higgs production, $p p \rightarrow h$, using MG5_aMC_v2_6_6[72], and then in PYTHIA6 decay the Higgs to a pair of LLPs (with $100 \%$ branching) and then these LLPs to quarks using similar coupling as in scenario (A). We study two different mass points which are less than half the Higgs mass $-10 \mathrm{GeV}$, and $30 \mathrm{GeV}$ having proper decay lengths of $10 \mathrm{~cm}$ and $100 \mathrm{~cm}$ each. The LLP is assumed to decay to light jets with $100 \%$ branching.

Table 5 shows the decay fractions in various detector parts for benchmark points of scenario $(\mathrm{C})$. In this scenario, the LLPs are very light and their boost is controlled by the difference in their mass and the mass of the Higgs boson. Therefore, we find that the $50 \mathrm{GeV}$ point has very less boost and decays mostly before the MTD.

We apply the BDT classification using track variables defined at L1 as discussed above and check their performance in classifying jets coming from benchmarks of scenario (C) and QCD background jets. The ROCs are shown in figure 13. The performance degrades than scenario (A) and scenario (B) benchmarks, as is expected, since these are very light LLPs and have very low jet multiplicities above our $p_{T}$ threshold of $60 \mathrm{GeV}$. Lowering the $p_{T}$ threshold requires taking into account QCD background with $p_{T}$ bins less than $50 \mathrm{GeV}$ at generation level, which again have huge cross sections and can increase the trigger rates to very high values. Also, the pile-up rates are high at lower $p_{T}$ values. The ROCs are 


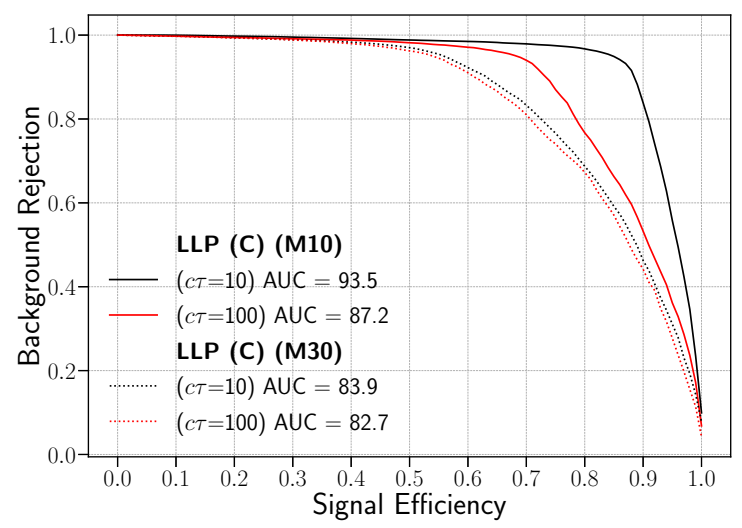

Figure 13. ROC curves for selecting displaced jets coming from LLP decay from prompt QCD ones for four benchmark points from scenario (C) with LLP masses $10 \mathrm{GeV}$ and $30 \mathrm{GeV}$ and decay lengths $10 \mathrm{~cm}$ and $100 \mathrm{~cm}$ using tracking variables.
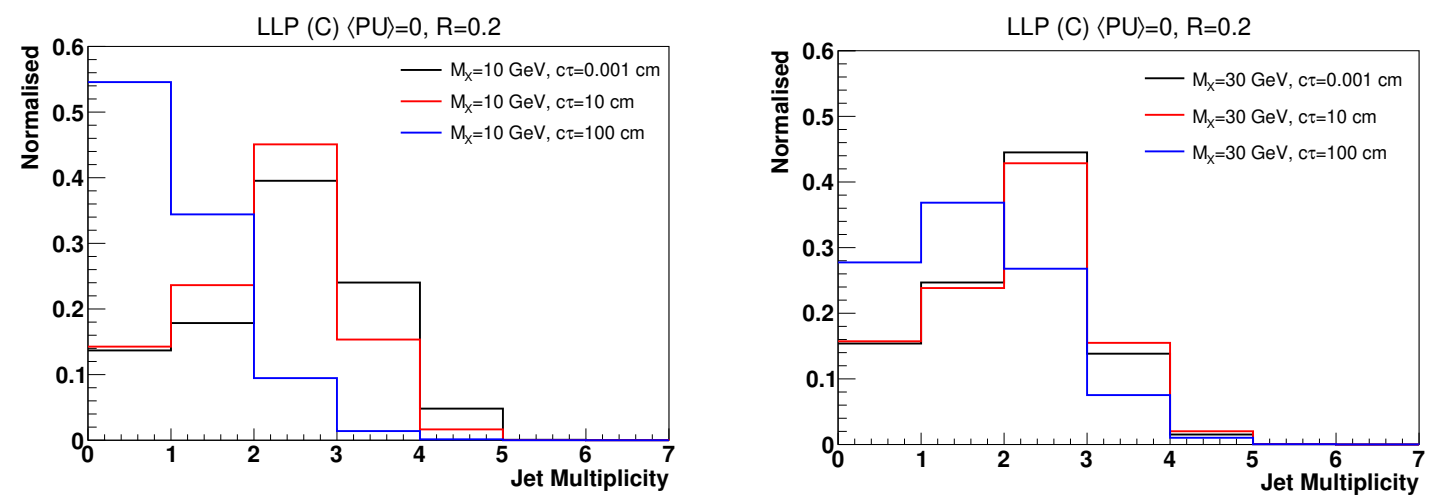

Figure 14. Distributions of jet multiplicity for LLPs of mass $10 \mathrm{GeV}$ (left) and $30 \mathrm{GeV}$ (right) with varying decay length.

almost flat till a signal efficiency of about $70-80 \%$ for LLP having a mass of $10 \mathrm{GeV}$, and we can still get reasonable QCD rejection $(\sim 95 \%)$ at these values of signal efficiencies, which will help in increasing sensitivity to lower LLP masses. For LLP mass of $30 \mathrm{GeV}$, there are even less chances of having jets with $p_{T}>60 \mathrm{GeV}$, and the performance also degrades.

Another observation is that the performance degrades faster from decay length $10 \mathrm{~cm}$ to $100 \mathrm{~cm}$ when the mass of the LLP is $10 \mathrm{GeV}$ than when it is $30 \mathrm{GeV}$. This is due to the fact that the $10 \mathrm{GeV}$ mass LLP has very high boost values due to its smaller mass which when has a large decay length, will mostly decay outside the HCAL, and hence, the jet multiplicity will be low. We compare in figure 14 the jet multiplicity distribution of the $10 \mathrm{GeV}$ (left) and $30 \mathrm{GeV}$ (right) LLPs with varying decay lengths. We observe that the jet multiplicity changes drastically for the $10 \mathrm{GeV}$ LLP from $10 \mathrm{~cm}$ decay length to $100 \mathrm{~cm}$, however, this change is not so drastic in the $30 \mathrm{GeV}$ LLP case.

We will now discuss the effect when an ISR jet is present in such a process. The ISR jet increases the boost of the LLPs, and we can get more $p_{T}>60 \mathrm{GeV}$ jets. However, an 


\begin{tabular}{|c|c|c|c|c|c|c|c|}
\hline $\begin{array}{c}\text { Mass }[\mathrm{GeV}], \\
\text { Decay Length }[\mathrm{cm}]\end{array}$ & $\begin{array}{c}\text { Reco as } \\
\text { L1 tracks }\end{array}$ & $\begin{array}{c}\text { Before } \\
\text { MTD }\end{array}$ & $\begin{array}{c}\text { Before } \\
\text { ECAL }\end{array}$ & $\begin{array}{c}\text { Before } \\
\text { HCAL }\end{array}$ & $\begin{array}{c}\text { Before } \\
\text { MS }\end{array}$ & $\begin{array}{c}\text { Inside } \\
\text { MS }\end{array}$ & $\begin{array}{c}\text { Outside } \\
\text { detector }\end{array}$ \\
\hline 10,10 & 2.27 & 71.63 & 3.20 & 7.14 & 8.33 & 4.83 & 2.61 \\
10,100 & 0.24 & 16.55 & 1.72 & 5.39 & 14.83 & 17.45 & 43.82 \\
30,10 & 6.53 & 88.21 & 1.07 & 1.80 & 1.39 & 0.81 & 0.18 \\
30,100 & 0.70 & 39.67 & 3.28 & 9.23 & 17.34 & 14.48 & 15.31 \\
50,10 & 12.90 & 85.34 & 0.49 & 0.58 & 0.44 & 0.21 & 0.03 \\
50,100 & 1.40 & 55.70 & 3.59 & 8.86 & 13.47 & 9.63 & 7.35 \\
\hline
\end{tabular}

Table 6. Fraction of decays for scenario (C) with an ISR jet of $p_{T}>50 \mathrm{GeV}$ in various detector parts.

\begin{tabular}{|c|c|c|}
\hline & $\begin{array}{c}\max \left(\sum p_{T}^{2} / n_{z_{a}}\right) \text { corresponds } \\
\text { to hard collision }\end{array}$ & $\frac{\sigma(g g \rightarrow h+1 j)}{\sigma(g g \rightarrow h)}$ \\
\hline$+0 j$ & $14.14 \%$ & - \\
$+1 j, p_{T}^{j}>50 \mathrm{GeV}$ & $68.26 \%$ & $25.73 \%$ \\
$+1 j, p_{T}^{j}>100 \mathrm{GeV}$ & $84.82 \%$ & $8.81 \%$ \\
$+1 j, p_{T}^{j}>150 \mathrm{GeV}$ & $93.54 \%$ & $3.76 \%$ \\
\hline
\end{tabular}

Table 7. Efficiency for correctly identifying the vertex corresponding to the hard collision, from where LLPs are produced, as the primary vertex (PV) at L1 for scenario (C) without and with an ISR jet, with increasing the minimum $p_{T}$ of the latter. Also quoted are the production cross sections and the fraction of this cross section to the total cross section, without any hard ISR jet.

increased boost will also mean increase in the decay length in the lab frame, and lesser number of jets within the first layer of the HCAL. Table 6 shows how these decay fractions get modified when the Higgs is produced with an ISR jet of $p_{T}>50 \mathrm{GeV}$.

The benefits of adding an ISR jet is that if the ISR jet is hard enough $\left(p_{T}>173 \mathrm{GeV}\right)$, it will help the event pass the single jet trigger. However, it comes with the cost of reduced cross section. As you increase the $p_{T}$ of the ISR jet, the cross section falls off drastically. The second column of table 7 shows how the efficiency of identifying the correct PV increases when the $p_{T}$ of the ISR jet is high, and the third column shows the decrease in the production cross section. To pass the single jet trigger, we need an ISR jet having at least $p_{T}>173 \mathrm{GeV}$, and from the last row of table 7 , we find that the cross section for such high $p_{T}$ jets is really small and we will only be able to trigger on $3.76 \%$ of such events.

We use the sample with an ISR jet of $p_{T}>50 \mathrm{GeV}$ to check how including the ISR affects the BDT performance. Figure 15 shows the BDT performances for the different benchmark points from scenario (C) when generated with an ISR jet using track variables. We find that the performance degrades when the event has an ISR jet in the ROCs. The 


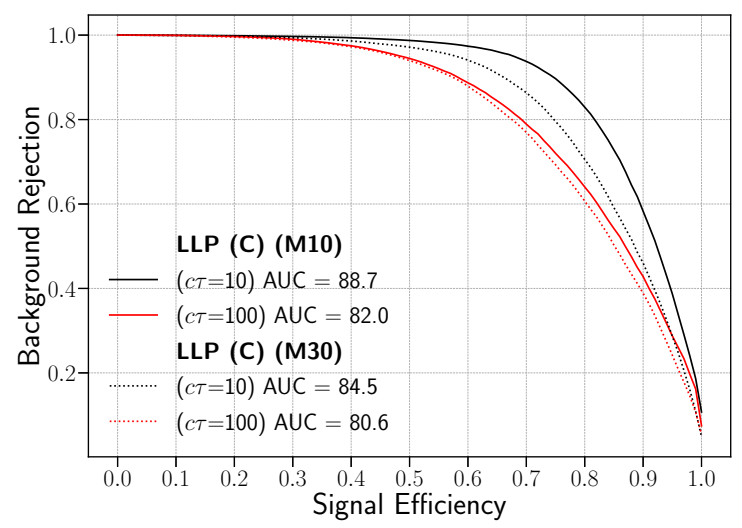

Figure 15. ROC curves for selecting displaced jets coming from LLP decay from prompt QCD ones for four benchmark points from scenario (C) with LLP masses $10 \mathrm{GeV}$ and $30 \mathrm{GeV}$ and decay lengths $10 \mathrm{~cm}$ and $100 \mathrm{~cm}$ using tracking variables when we generate the LLP processes with an initial state radiated jet having $p_{T}>50 \mathrm{GeV}$.

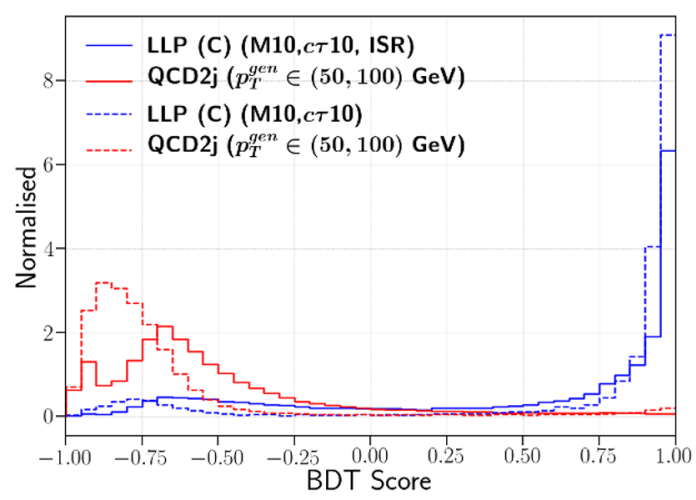

Figure 16. Distribution of BDT scores when trained using track variables for prompt and displaced jets from an LLP with mass $10 \mathrm{GeV}$ and decay length $10 \mathrm{~cm}$ produced with and without an ISR jet (scenario $(\mathrm{C})$ ).

reason could be that the ISR jet resembles the prompt QCD jet, which is our background and therefore, the training is affected. This can be seen in figure 16 where we have compared the BDT score distributions for the signal and background when trained with LLP benchmark point from scenario (C) where mass and decay length of the LLP is $10 \mathrm{GeV}$ and $10 \mathrm{~cm}$ respectively, generated with and without the ISR. We find that with ISR the signal BDT scores are slightly more towards the background BDT score values than that without ISR.

\subsection{Scenario where LLPs are produced from the decay of a heavy resonance and decay to jets}

The LLPs can also be produced from the decay of a heavy resonance which could be a new BSM particle. We consider such a case where the mass of the intermediate resonance is $300 \mathrm{GeV}$ and the LLPs which come from its decay have a mass of $10 \mathrm{GeV}$ and decay length 

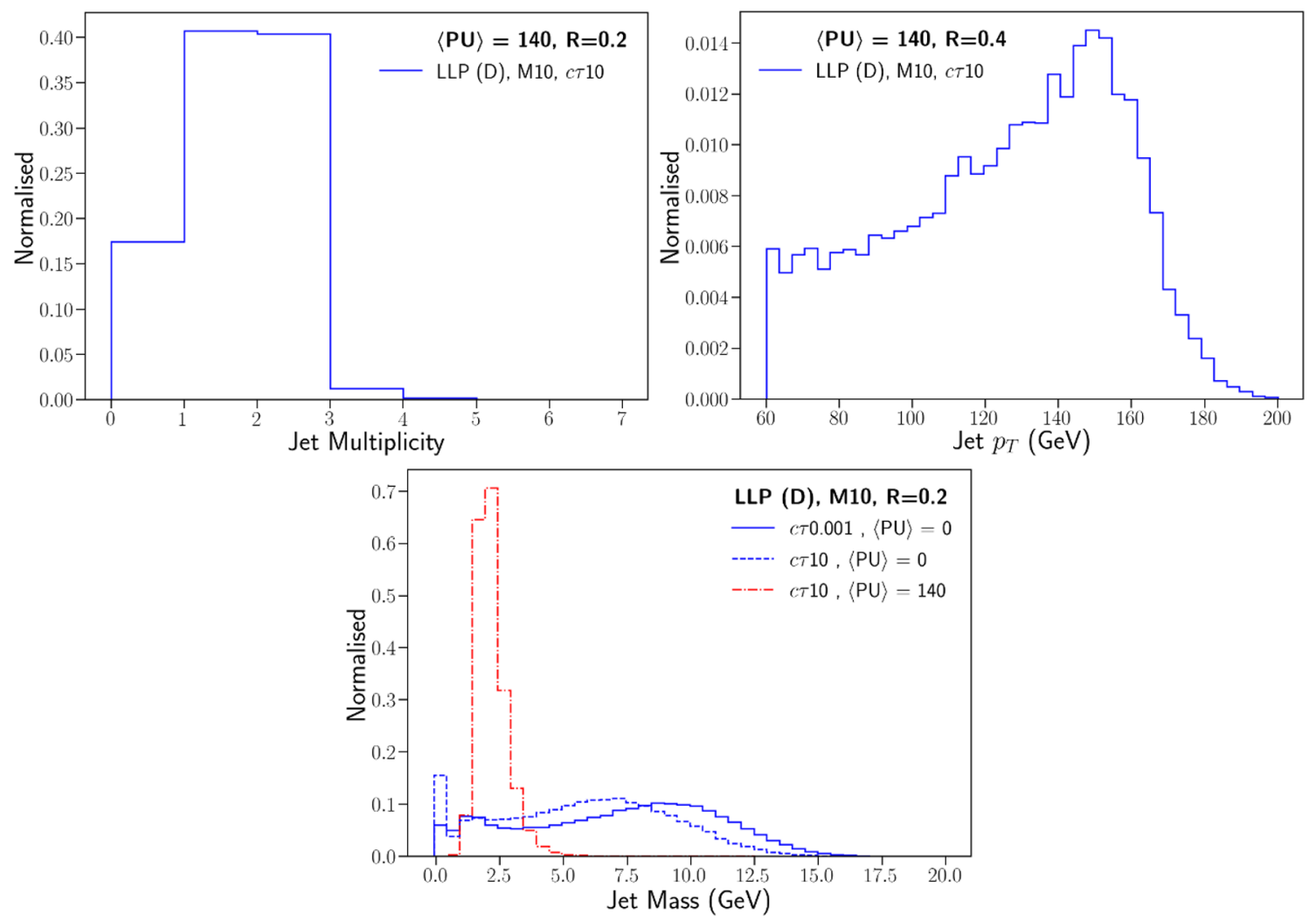

Figure 17. Top: jet multiplicity, jet $p_{T}$, and bottom: jet mass distribution for the case when LLPs (of mass $10 \mathrm{GeV}$ and decay length $10 \mathrm{~cm}$ ) come from the decay of a heavy resonance of mass $300 \mathrm{GeV}$.

of $10 \mathrm{~cm}$, and this is denoted as scenario (D). The LLPs in this case will be highly boosted since they are light and come from the decay of a heavy particle.

Figure 17 shows the multiplicity, $p_{T}$ and jet mass distributions of jets coming from an LLP of mass $10 \mathrm{GeV}$ and decay length $10 \mathrm{~cm}$, which comes from the decay of a $300 \mathrm{GeV}$ resonance. As we discussed earlier, in this case the LLPs will be highly boosted, which will increase their decay length in the lab frame. Therefore, the decay products can be close enough to get identified as a single jet. As we can see from the multiplicity plot, most of the time the multiplicity is one or two, which is due to increased probability of merging of two jets coming from the LLP decay.

The jet $p_{T}$ peaks around $150 \mathrm{GeV}$, which is half the heavy resonance mass, and in most cases the $p_{T}$ of the LLP will be close to this value. This implies that both the jets coming from the LLP decay is identified as a single jet. We therefore, expect a peak around $10 \mathrm{GeV}$ in the jet mass distribution. In case of no displacement, we do find a small peak around that value, since the jets are boosted enough that they can be contained within $R=0.2$ in some cases, even with very low $c \tau$ value. However, with increasing $c \tau$, we do not find any resonance peak around $10 \mathrm{GeV}$. This is due to the mismatch of the jets' actual $\eta-\phi$ with the one that is measured in the detector, which gives a wrong estimate of the jet mass. 
We also notice an important feature that with increasing $c \tau$ the jet mass is mostly close to zero, which happens when the LLP is displaced enough such that all its constituents fall within a single tower, and the mass of a single tower is close to zero. However, with the addition of $140 \mathrm{PU}$ events, this effect goes away.

The jet $p_{T}$ for this scenario is mostly on the higher side and can therefore, pass the standard single jet trigger. We therefore, do not present our analysis based on tracking at L1 for this scenario.

\section{Conclusions and outlook}

Long-lived particles, strongly motivated from many BSM theories, that travel some distance in the detector before decaying to SM particles, provide a compelling substitute to prompt hypothetical particles, especially, considering the absence of any discovery of BSM particles at the current LHC experiments. Models including LLPs could address important questions still unresolved by the SM. Unique signatures of LLPs offer staggering scope for discovery of physics beyond the standard model in the LHC experiments, but simultaneously they require specific and dedicated triggering strategies since they can be easily missed. Since their production cross sections depend on various model parameters, they can also have really small values, and in that case, we need to ensure that the triggers are efficient enough so that we don't miss them even at the first level.

The LHC experiments are going through a significant Phase II upgrade scheme to get ready for the gruelling conditions of HL-LHC. For CMS, the increase of instantaneous luminosity at the HL-LHC will necessitate the introduction of tracker information, for the first time, in the level-1 trigger system to retain an acceptable trigger rate.

In this work we have shown that the usual level-1 triggers targeting prompt particles, will be inefficient for LLP searches in some scenarios, for example, when light LLPs are pair-produced directly and decay into jets, in the high PU environment of HL-LHC. This points to the need to develop dedicated L1 triggers for LLP searches. In our paper, we have presented the studies in the context of CMS detector and its upgrade in HL-LHC, however, all the ideas should be qualitatively valid for ATLAS detector as well.

We begin by studying how the jet distributions of both signal and background events change in the high PU scenarios, and how this effect can be minimised by considering narrow jets. For LLPs as signal, considering narrow jets do not affect the jet distributions as much as it affects the background jets, since displaced jets are physically more contained in smaller regions. However, this is not enough to suppress the huge QCD background and one has to use other features of displaced jets to differentiate them from the prompt ones.

In Phase-II the FPGAs used in L1 trigger hardware will be sophisticated enough to handle small scale machine learning (ML) applications. In the context of the L1 track triggers, one of the most important upgrades of CMS experiment in Phase-II of LHC, we have explored the performance of CMS in differentiating between prompt and displaced jets, the former coming from QCD dijet background and the latter from LLP decays. We have constructed several variables related to L1 tracks, and used them to train boosted decision trees. Our study has shown that it is possible to develop some dedicated LLP 
triggers based on the BDT classification which can help increase the trigger efficiency of events with LLPs and reasonably moderate background rates, even when we lower the $p_{T}$ threshold.

We have also presented a discussion on some other LLP scenarios. We have showed that when the LLP decays to some invisible particles in addition to jets, the $\mathbb{E}_{T}$ distribution as calculated using the L1 tracks will change completely when the decay length of the LLP is increased, and therefore, triggers based on $\mathbb{E}_{T}$ won't be efficient. However, the track based triggers have promising results in selecting such events. We have also discussed the effect of an ISR jet produced in association with light LLPs on the trigger efficiencies.

We conclude that using variables related to track will greatly improve the capability to trigger on LLPs in L1 triggering stage in the high PU environment of HL-LHC. We have studied in detail how one can utilise these to develop dedicated BDT based triggers for LLPs. One has to explore how more complex avenues of ML tools can be implemented at the L1 trigger and how they can improve the performance of dedicated LLP triggers, with our results as the starting point.

\section{Acknowledgments}

The work of B.B. is supported by the Department of Science and Technology, Government of India, under Grant No. IFA13- PH-75 (INSPIRE Faculty Award). The work of S.M. is supported by the German Federal Ministry of Education and Research BMBF. R.S. and P.S. would like to thank Rahool Kumar Barman and Amit Adhikary for useful discussions. 


\section{A Supplementary materials in developing the track-based L1 trigger}

\section{A.1 Distributions of tracking variables}
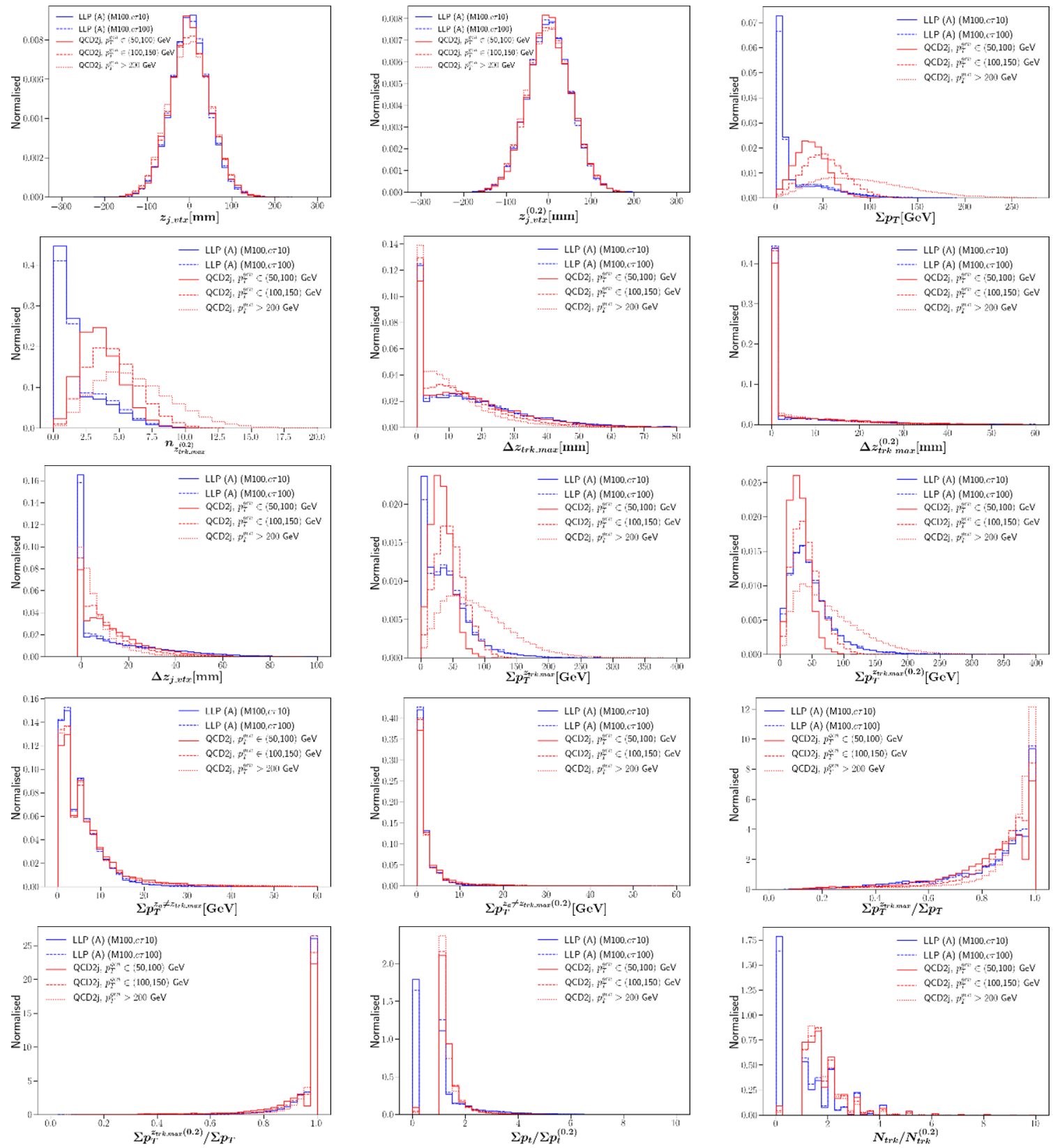

Figure 18. Distributions for the rest of the track variables for two signal benchmark points from scenario (A) and QCD dijet processes with different $p_{T}$ cuts at the parton level. 

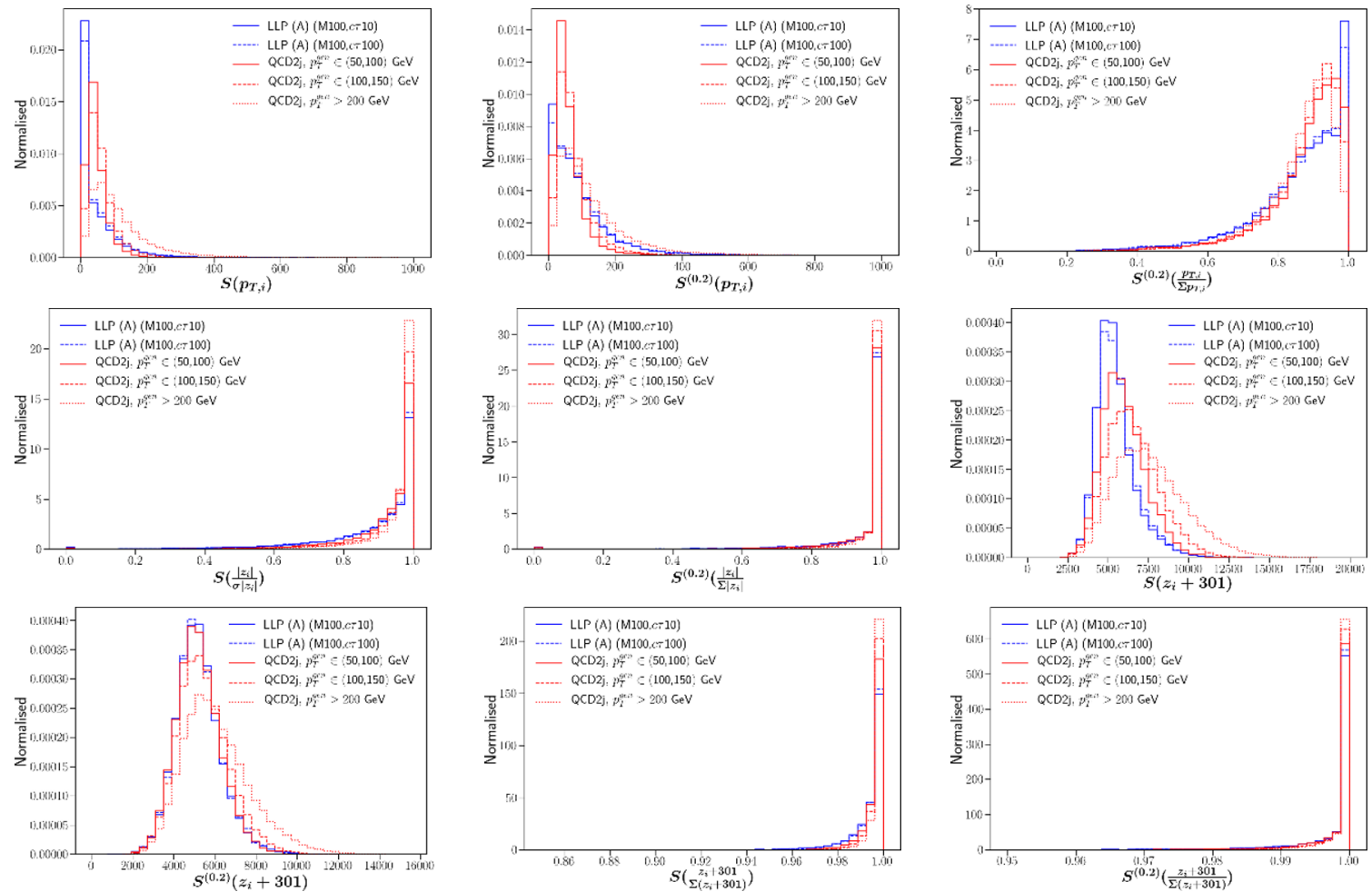

Figure 19. Distributions for the rest of the track variables (contd.) for two signal benchmark points from scenario (A) and QCD dijet processes with different $p_{T}$ cuts at the parton level. 


\section{A.2 Correlation matrix of variables}

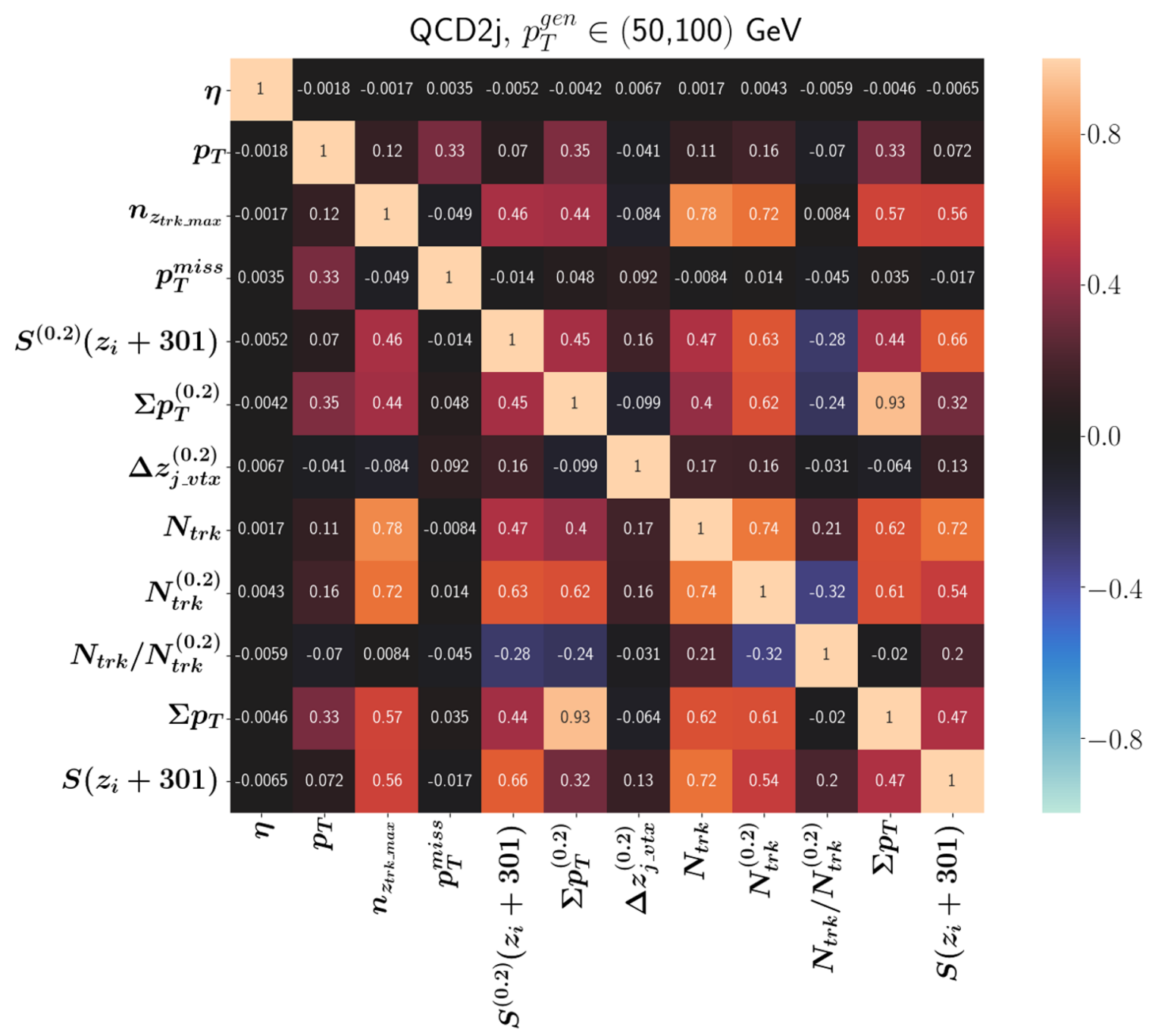

Figure 20. Correlation matrices of background jets from QCD dijet events with $p_{T} \in(50,100) \mathrm{GeV}$ for variables using L1 tracking. 


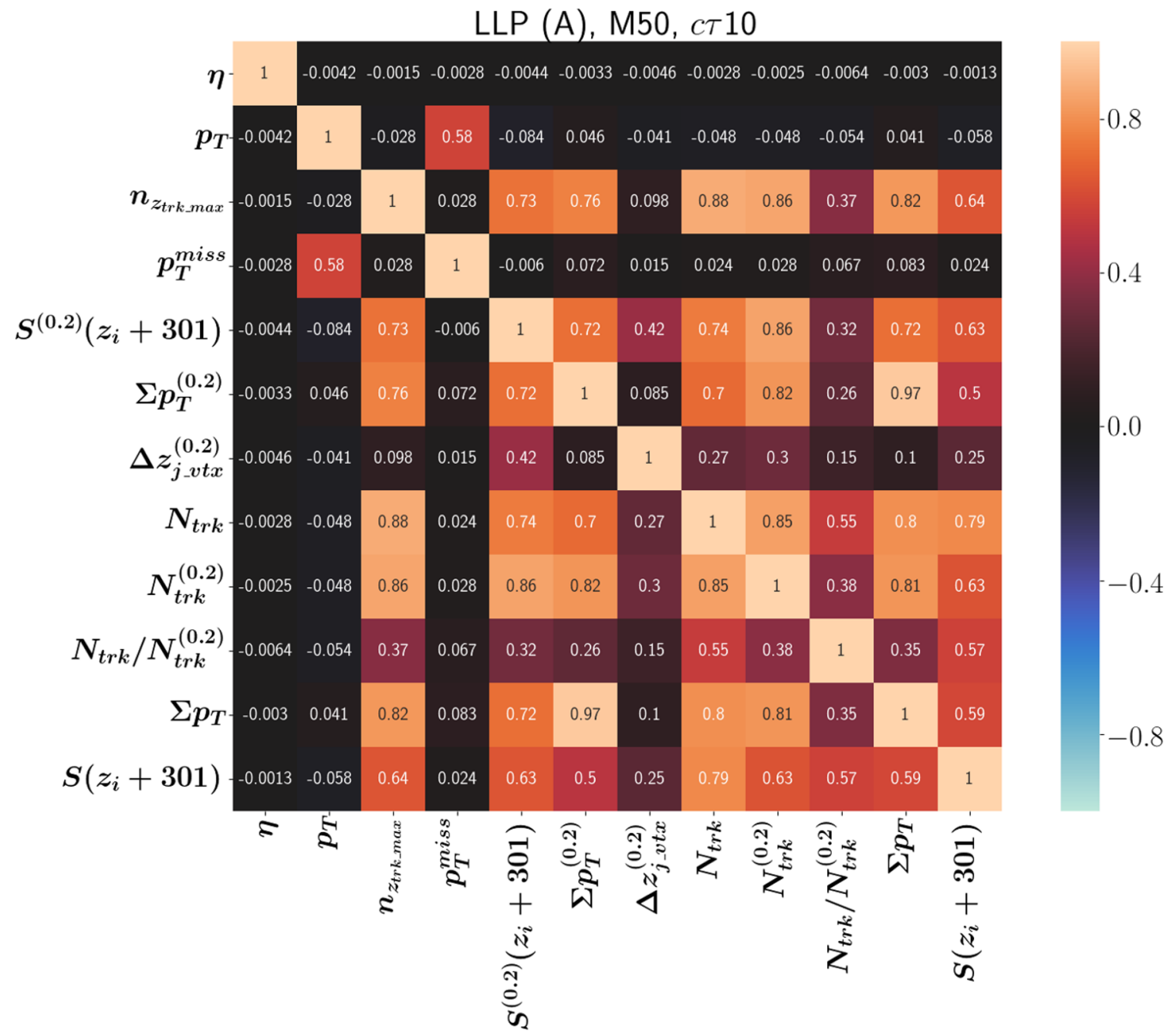

Figure 21. Correlation matrices of signal jets from LLP benchmark point where mass and decay length of the LLP is $50 \mathrm{GeV}$ and $10 \mathrm{~cm}$ respectively from scenario (A) for variables using both L1 tracking. 
A.3 Trigger efficiencies and rates for $90 \%$ background rejection

\begin{tabular}{|c|c|c|c|c|c|}
\hline LLP (A) & $\begin{array}{c}\text { QCD } 2 \mathrm{j} \\
p_{T}^{\text {gen }}[\mathrm{GeV}] \\
\left(\mathcal{R}_{B}[\mathrm{kHz}]\right)\end{array}$ & $\begin{array}{c}T_{2}^{1} \\
\mathcal{R}_{B}[\mathrm{kHz}] \\
\left(\epsilon_{S}[\%]\right)\end{array}$ & $\begin{array}{c}T_{3}^{1} \\
\mathcal{R}_{B}[\mathrm{kHz}] \\
\left(\epsilon_{S}[\%]\right)\end{array}$ & $\begin{array}{c}T_{41}^{1} \\
\mathcal{R}_{B}[\mathrm{kHz}] \\
\left(\epsilon_{S}[\%]\right)\end{array}$ & $\begin{array}{c}T_{42}^{1} \\
\mathcal{R}_{B}[\mathrm{kHz}] \\
\left(\epsilon_{S}[\%]\right)\end{array}$ \\
\hline \multirow{4}{*}{$\begin{array}{c}M=50 \mathrm{GeV} \\
c \tau=10 \mathrm{~cm}\end{array}$} & $50,100(1046)$ & $34.7(17.41)$ & $31.6(17.07)$ & $25.2(14.47)$ & $29.8(15.84)$ \\
\hline & $100,150(53.4)$ & $6.7(17.02)$ & $5.3(16.71)$ & $1.5(14.21)$ & $3.0(15.47)$ \\
\hline & $150,200(7.5)$ & $1.2(16.28)$ & $0.9(16.01)$ & $0.1(13.61)$ & $0.14(14.77)$ \\
\hline & $>200(2.7)$ & $0.5(15.76)$ & $0.2(15.53)$ & $0.02(13.12)$ & $0.03(14.29)$ \\
\hline \multirow{4}{*}{$\begin{aligned} M & =50 \mathrm{GeV} \\
c \tau & =100 \mathrm{~cm}\end{aligned}$} & $50,100(1046)$ & $34.7(12.32)$ & $31.6(11.98)$ & $25.2(9.63)$ & $29.8(10.71)$ \\
\hline & $100,150(53.4)$ & $6.7(11.90)$ & $5.3(11.59)$ & $1.5(9.39)$ & $3(10.34)$ \\
\hline & $150,200(7.5)$ & $1.2(11.14)$ & $0.9(10.89)$ & $0.1(8.89)$ & $0.14(9.72)$ \\
\hline & $>200(2.7)$ & $0.5(10.73)$ & $0.2(10.51)$ & $0.02(8.57)$ & $0.03(9.39)$ \\
\hline \multirow{4}{*}{$\begin{array}{c}M=100 \mathrm{GeV} \\
c \tau=10 \mathrm{~cm}\end{array}$} & $50,100(1046)$ & $34.4(72.44)$ & $30.3(68.91)$ & $22.1(46.69)$ & $28.3(57.03)$ \\
\hline & $100,150(53.4)$ & $6.7(68.23)$ & $4.7(65.74)$ & $0.9(43.52)$ & $2.1(52.99)$ \\
\hline & $150,200(7.5)$ & $1.2(60.95)$ & $0.8(59.39)$ & $0.05(36.21)$ & $0.08(45.70)$ \\
\hline & $>200(2.7)$ & $0.5(51.47)$ & $0.2(50.33)$ & $0.0(25.37)$ & $0.01(35.45)$ \\
\hline \multirow{4}{*}{$\begin{array}{c}M=100 \mathrm{GeV} \\
c \tau=100 \mathrm{~cm}\end{array}$} & $50,100(1046)$ & $34.4(58.99)$ & $30.3(55.49)$ & $22.1(34.22)$ & $28.3(42.70)$ \\
\hline & $100,150(53.4)$ & $6.7(54.64)$ & 4.7 (51.98) & $0.9(31.50)$ & $2.1(38.72)$ \\
\hline & $150,200(7.5)$ & $1.2(47.58)$ & $0.8(45.68)$ & $0.05(25.55)$ & $0.08(32.40)$ \\
\hline & $>200(2.7)$ & $0.5(38.98)$ & $0.2(37.51)$ & $0.0(17.12)$ & $0.01(24.16)$ \\
\hline
\end{tabular}

Table 8. Efficiency of selecting QCD and LLP events for scenario (A) benchmark points with the modified trackless trigger at L1 for Phase-II. BDT cut is applied considering $90 \%$ background rejection. Quantity in parenthesis represents corresponding signal efficiency. 


\section{A.4 The 200 PU scenario}

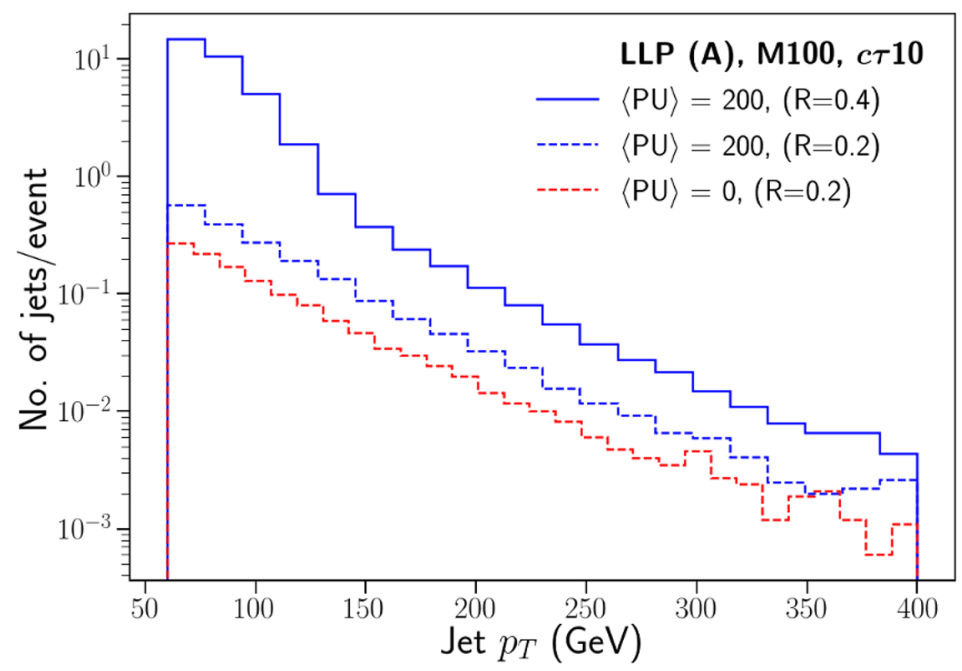

Figure 22. Jet $p_{T}$ distribution for jets clustered using anti- $k_{T}$ with $R=0.2$ and $R=0.4$ coming from LLP benchmark $\left(M_{X}=100 \mathrm{GeV}\right.$ and $\left.c \tau=10 \mathrm{~cm}\right)$ from scenario (A) with 200 PU compared with the zero PU jet $p_{T}$ distribution.

Open Access. This article is distributed under the terms of the Creative Commons Attribution License (CC-BY 4.0), which permits any use, distribution and reproduction in any medium, provided the original author(s) and source are credited.

\section{References}

[1] L. Lee, C. Ohm, A. Soffer and T.-T. Yu, Collider Searches for Long-Lived Particles Beyond the Standard Model, Prog. Part. Nucl. Phys. 106 (2019) 210 [arXiv:1810.12602] [InSPIRE].

[2] G.F. Giudice and R. Rattazzi, Theories with gauge mediated supersymmetry breaking, Phys. Rept. 322 (1999) 419 [hep-ph/9801271] [INSPIRE].

[3] G.F. Giudice and A. Romanino, Split supersymmetry, Nucl. Phys. B 699 (2004) 65 [Erratum ibid. 706 (2005) 487] [hep-ph/0406088] [INSPIRE].

[4] R. Barbier et al., R-parity violating supersymmetry, Phys. Rept. 420 (2005) 1 [hep-ph/0406039] [INSPIRE].

[5] N. Arkani-Hamed, S. Dimopoulos, G.F. Giudice and A. Romanino, Aspects of split supersymmetry, Nucl. Phys. B 709 (2005) 3 [hep-ph/0409232] [INSPIRE].

[6] G. Burdman, Z. Chacko, H.-S. Goh and R. Harnik, Folded supersymmetry and the LEP paradox, JHEP 02 (2007) 009 [hep-ph/0609152] [INSPIRE].

[7] S.P. de Alwis, On Anomaly Mediated SUSY Breaking, Phys. Rev. D 77 (2008) 105020 [arXiv: 0801.0578] [INSPIRE].

[8] P. Meade, M. Reece and D. Shih, Long-Lived Neutralino NLSPs, JHEP 10 (2010) 067 [arXiv: 1006.4575] [INSPIRE]. 
[9] S. Biswas, J. Chakrabortty and S. Roy, Multi-photon signal in supersymmetry comprising non-pointing photon(s) at the LHC, Phys. Rev. D 83 (2011) 075009 [arXiv:1010.0949] [INSPIRE].

[10] J. Fan, M. Reece and J.T. Ruderman, Stealth Supersymmetry, JHEP 11 (2011) 012 [arXiv:1105.5135] [INSPIRE].

[11] M. Ibe, S. Matsumoto and T.T. Yanagida, Pure Gravity Mediation with $m_{3 / 2}=10-100 \mathrm{TeV}$, Phys. Rev. D 85 (2012) 095011 [arXiv: 1202.2253] [InSPIRE].

[12] P.W. Graham, D.E. Kaplan, S. Rajendran and P. Saraswat, Displaced Supersymmetry, JHEP 07 (2012) 149 [arXiv: 1204.6038] [INSPIRE].

[13] B. Bhattacherjee, B. Feldstein, M. Ibe, S. Matsumoto and T.T. Yanagida, Pure gravity mediation of supersymmetry breaking at the Large Hadron Collider, Phys. Rev. D 87 (2013) 015028 [arXiv: 1207.5453] [inSPIRE].

[14] A. Arvanitaki, N. Craig, S. Dimopoulos and G. Villadoro, Mini-Split, JHEP 02 (2013) 126 [arXiv: 1210.0555] [INSPIRE].

[15] D.G. Cerdeño, V. Martín-Lozano and O. Seto, Displaced vertices and long-lived charged particles in the NMSSM with right-handed sneutrinos, JHEP 05 (2014) 035 [arXiv: 1311.7260] [INSPIRE].

[16] K. Rolbiecki and K. Sakurai, Long-lived bino and wino in supersymmetry with heavy scalars and higgsinos, JHEP 11 (2015) 091 [arXiv: 1506.08799] [INSPIRE].

[17] S. Banerjee, G. Bélanger, A. Ghosh and B. Mukhopadhyaya, Long-lived stau, sneutrino dark matter and right-slepton spectrum, JHEP 09 (2018) 143 [arXiv: 1806.04488] [INSPIRE].

[18] D. Dercks, J. De Vries, H.K. Dreiner and Z.S. Wang, R-parity Violation and Light Neutralinos at CODEX-b, FASER, and MATHUSLA, Phys. Rev. D 99 (2019) 055039 [arXiv: 1810.03617] [INSPIRE].

[19] H. Fukuda, N. Nagata, H. Oide, H. Otono and S. Shirai, Cornering Higgsinos Using Soft Displaced Tracks, Phys. Rev. Lett. 124 (2020) 101801 [arXiv:1910.08065] [INSPIRE].

[20] L.J. Hall, K. Jedamzik, J. March-Russell and S.M. West, Freeze-In Production of FIMP Dark Matter, JHEP 03 (2010) 080 [arXiv:0911.1120] [INSPIRE].

[21] D. Tucker-Smith and N. Weiner, Inelastic dark matter, Phys. Rev. D 64 (2001) 043502 [hep-ph/0101138] [INSPIRE].

[22] R.T. Co, F. D'Eramo, L.J. Hall and D. Pappadopulo, Freeze-In Dark Matter with Displaced Signatures at Colliders, JCAP 12 (2015) 024 [arXiv:1506.07532] [INSPIRE].

[23] A.G. Hessler, A. Ibarra, E. Molinaro and S. Vogl, Probing the scotogenic FIMP at the LHC, JHEP 01 (2017) 100 [arXiv:1611.09540] [INSPIRE].

[24] V.V. Khoze, A.D. Plascencia and K. Sakurai, Simplified models of dark matter with a long-lived co-annihilation partner, JHEP 06 (2017) 041 [arXiv:1702.00750] [INSPIRE].

[25] G. Bélanger et al., LHC-friendly minimal freeze-in models, JHEP 02 (2019) 186 [arXiv: 1811.05478] [INSPIRE].

[26] A. Goudelis, K.A. Mohan and D. Sengupta, Clockworking FIMPs, JHEP 10 (2018) 014 [arXiv: 1807.06642] [INSPIRE].

[27] K.J. Bae, M. Park and M. Zhang, Demystifying freeze-in dark matter at the LHC, Phys. Rev. D 101 (2020) 115036 [arXiv: 2001.02142] [INSPIRE]. 
[28] M.J. Strassler and K.M. Zurek, Echoes of a hidden valley at hadron colliders, Phys. Lett. B 651 (2007) 374 [hep-ph/0604261] [INSPIRE].

[29] M.J. Strassler and K.M. Zurek, Discovering the Higgs through highly-displaced vertices, Phys. Lett. B 661 (2008) 263 [hep-ph/0605193] [INSPIRE].

[30] M.J. Strassler, Possible effects of a hidden valley on supersymmetric phenomenology, hep-ph/0607160 [INSPIRE].

[31] D. Curtin et al., Long-Lived Particles at the Energy Frontier: The MATHUSLA Physics Case, Rept. Prog. Phys. 82 (2019) 116201 [arXiv:1806.07396] [InSPIRE].

[32] ATLAS collaboration, Search for nonpointing and delayed photons in the diphoton and missing transverse momentum final state in $8 \mathrm{TeV}$ pp collisions at the LHC using the ATLAS detector, Phys. Rev. D 90 (2014) 112005 [arXiv: 1409.5542] [INSPIRE].

[33] ATLAS collaboration, Search for long-lived charginos based on a disappearing-track signature in pp collisions at $\sqrt{s}=13 \mathrm{TeV}$ with the ATLAS detector, JHEP 06 (2018) 022 [arXiv: 1712.02118] [INSPIRE].

[34] ATLAS collaboration, Search for long-lived, massive particles in events with displaced vertices and missing transverse momentum in $\sqrt{s}=13 \mathrm{TeV} p p$ collisions with the ATLAS detector, Phys. Rev. D 97 (2018) 052012 [arXiv: 1710.04901] [INSPIRE].

[35] ATLAS collaboration, Search for light long-lived neutral particles produced in pp collisions at $\sqrt{s}=13 \mathrm{TeV}$ and decaying into collimated leptons or light hadrons with the ATLAS detector, Eur. Phys. J. C 80 (2020) 450 [arXiv: 1909.01246] [InSPIRE].

[36] ATLAS collaboration, Search for long-lived neutral particles in pp collisions at $\sqrt{s}=13 \mathrm{TeV}$ that decay into displaced hadronic jets in the ATLAS calorimeter, Eur. Phys. J. C 79 (2019) 481 [arXiv: 1902.03094] [INSPIRE].

[37] ATLAS collaboration, Search for displaced vertices of oppositely charged leptons from decays of long-lived particles in pp collisions at $\sqrt{s}=13 \mathrm{TeV}$ with the ATLAS detector, Phys. Lett. $B 801$ (2020) 135114 [arXiv: 1907.10037] [INSPIRE].

[38] CMS collaboration, Search for long-lived neutral particles in the final state of delayed photons and missing energy in proton-proton collisions at $\sqrt{s}=8 \mathrm{TeV}$, CMS-PAS-EXO-12-035 (2015) [INSPIRE].

[39] CMS collaboration, Search for long-lived charged particles in proton-proton collisions at $\sqrt{s}=13$ TeV, Phys. Rev. D 94 (2016) 112004 [arXiv:1609.08382] [INSPIRE].

[40] CMS collaboration, Search for long-lived particles using delayed jets and missing transverse momentum with proton-proton collisions at $\sqrt{s}=13$ TeV, CMS-PAS-EXO-19-001 (2019) [INSPIRE].

[41] CMS collaboration, Search for new long-lived particles at $\sqrt{s}=13$ TeV, Phys. Lett. B 780 (2018) 432 [arXiv: 1711.09120] [INSPIRE].

[42] CMS collaboration, Search for decays of stopped exotic long-lived particles produced in proton-proton collisions at $\sqrt{s}=13 \mathrm{TeV}$, JHEP 05 (2018) 127 [arXiv:1801.00359] [INSPIRE].

[43] CMS collaboration, Search for long-lived particles using nonprompt jets and missing transverse momentum with proton-proton collisions at $\sqrt{s}=13$ TeV, Phys. Lett. B 797 (2019) 134876 [arXiv:1906.06441] [INSPIRE]. 
[44] CMS collaboration, Search for long-lived particles using delayed photons in proton-proton collisions at $\sqrt{s}=13$ TeV, Phys. Rev. D 100 (2019) 112003 [arXiv:1909.06166] [InSPIRE].

[45] J. Alimena et al., Searching for Long-Lived Particles beyond the Standard Model at the Large Hadron Collider, arXiv: 1903.04497 [INSPIRE].

[46] S. Banerjee, G. Bélanger, B. Bhattacherjee, F. Boudjema, R.M. Godbole and S. Mukherjee, Novel signature for long-lived particles at the LHC, Phys. Rev. D 98 (2018) 115026 [arXiv: 1706.07407] [INSPIRE].

[47] B. Bhattacherjee, S. Mukherjee and R. Sengupta, Study of energy deposition patterns in hadron calorimeter for prompt and displaced jets using convolutional neural network, JHEP 11 (2019) 156 [arXiv: 1904.04811] [INSPIRE].

[48] ATLAS collaboration, Triggers for displaced decays of long-lived neutral particles in the ATLAS detector, 2013 JINST 8 P07015 [arXiv: 1305.2284] [INSPIRE].

[49] ATLAS collaboration, Search for long-lived particles produced in pp collisions at $\sqrt{s}=13$ TeV that decay into displaced hadronic jets in the ATLAS muon spectrometer, Phys. Rev. D 99 (2019) 052005 [arXiv: 1811.07370] [INSPIRE].

[50] G. Apollinari, O. Brüning, T. Nakamoto and L. Rossi, High Luminosity Large Hadron Collider HL-LHC, in High-Luminosity Large Hadron Collider. Preliminary Design Report, CERN Yellow Reports 1, G. Apollinari, I. Béjar Alonso, O. Brüning, M. Lamont and L. Rossi eds., CERN, Geneva Switzerland (2015), chapter 1 [CERN-2015-005] [arXiv: 1705.08830] [INSPIRE].

[51] J. Butler, D. Contardo, M. Klute, J. Mans and L. Silvestris, Technical Proposal for the Phase-II Upgrade of the CMS Detector, CERN-LHCC-2015-010 (2015) [LHCC-P-008] [CMS-TDR-15-02] [INSPIRE].

[52] T. James, A hardware track-trigger for CMS at the High Luminosity LHC, CMS-TS-2018-025 (2018) [CERN-THESIS-2018-241].

[53] M. Mårtensson, M. Isacson, H. Hahne, R. Gonzalez Suarez and R. Brenner, To catch a long-lived particle: hit selection towards a regional hardware track trigger implementation, 2019 JINST 14 P11009 [arXiv:1907.09846] [INSPIRE].

[54] Y. Gershtein, CMS Hardware Track Trigger: New Opportunities for Long-Lived Particle Searches at the HL-LHC, Phys. Rev. D 96 (2017) 035027 [arXiv:1705.04321] [InSPIRE].

[55] CMS collaboration, The Phase-2 Upgrade of the CMS Endcap Calorimeter, CERN-LHCC-2017-023 (2017) [CMS-TDR-019] [INSPIRE].

[56] M. Cacciari, G.P. Salam and G. Soyez, The anti- $k_{t}$ jet clustering algorithm, JHEP 04 (2008) 063 [arXiv:0802.1189] [INSPIRE].

[57] A. Zabi et al., Triggering on electrons, jets and tau leptons with the CMS upgraded calorimeter trigger for the LHC RUN II, 2016 JINST 11 C02008 [INSPIRE].

[58] V. Rekovic CMS Trigger @ HL LHC, in proceedings of the TDII-2018: Second Workshop on Triggering Discoveries in High Energy Physics, Puebla, Mexico, 29 January-2 February 2018 and online pdf version at https://indico.cern.ch/event/659612/contributions/2836319/ attachments/1593933/2525755/CMSTrigger-HL-LHC-Puebla_updated.pdf.

[59] J. Jones-Pérez, J. Masias and J.D. Ruiz-Álvarez, Search for Long-Lived Heavy Neutrinos at the LHC with a VBF Trigger, Eur. Phys. J. C 80 (2020) 642 [arXiv: 1912.08206] [INSPIRE]. 
[60] T. Sjöstrand, S. Mrenna and P.Z. Skands, PYTHIA 6.4 Physics and Manual, JHEP 05 (2006) 026 [hep-ph/0603175] [INSPIRE].

[61] DELPHES 3 collaboration, DELPHES 3, A modular framework for fast simulation of a generic collider experiment, JHEP 02 (2014) 057 [arXiv: 1307.6346] [INSPIRE].

[62] CMS collaboration, CMS Physics: Technical Design Report Volume 1: Detector Performance and Software, CERN-LHCC-2006-001 (2006) [INSPIRE].

[63] CMS collaboration, Technical proposal for a mip timing detector in the CMS experiment Phase 2 upgrade, CERN-LHCC-2017-027 (2017) [LHCC-P-009].

[64] S. Banerjee, B. Bhattacherjee, A. Goudelis, B. Herrmann, D. Sengupta and R. Sengupta, Determining the lifetime of long-lived particles at the LHC, arXiv:1912.06669 [INSPIRE].

[65] T. Sjöstrand, S. Mrenna and P.Z. Skands, A Brief Introduction to PYTHIA 8.1, Comput. Phys. Commun. 178 (2008) 852 [arXiv:0710.3820] [INSPIRE].

[66] ATLAS collaboration, The ATLAS Tau Trigger in Run 2, ATLAS-CONF-2017-061 (2017) [INSPIRE].

[67] https://docs.scipy.org/doc/scipy/reference/generated/scipy.stats.entropy.html.

[68] A. Hocker et al., TMVA - Toolkit for Multivariate Data Analysis, physics/0703039 [INSPIRE].

[69] R. Brun and F. Rademakers, ROOT: An object oriented data analysis framework, Nucl. Instrum. Meth. A 389 (1997) 81 [INSPIRE].

[70] J. Duarte et al., Fast inference of deep neural networks in FPGAs for particle physics, 2018 JINST 13 P07027 [arXiv: 1804.06913] [INSPIRE].

[71] S. Summers et al., Fast inference of Boosted Decision Trees in FPGAs for particle physics, 2020 JINST 15 P05026 [arXiv:2002.02534] [INSPIRE].

[72] J. Alwall, M. Herquet, F. Maltoni, O. Mattelaer and T. Stelzer, MadGraph 5: Going Beyond, JHEP 06 (2011) 128 [arXiv: 1106.0522] [INSPIRE]. 